\title{
Direct measurements of polymer-induced forces
}

\author{
Dzina Kleshchanok*, Remco Tuinier, Peter R. Lang
}

Forschungszentrum Jülich, Institut für Festkörperforschung, Weiche Materie, 52425 Jülich, Germany

E-mail: D.Kleshchanok@fz-juelich.de

\begin{abstract}
Colloid-polymer mixtures are found in dispersions that are an important part of people's everyday life. The dynamics and phase stability of colloid-polymer mixtures depend on the interactions that are present in these systems. Therefore, knowledge of interactions is of basic interest. Depending on their adsorption affinity polymers added to the colloidal suspension can cause steric stabilization or flocculation due to depletion or adsorption (bridging). This paper reviews theoretical and experimental work performed on polymer-induced interactions in colloidal suspensions. Theoretically, polymers were mainly treated as ideal flexible chains or even generalized as non-interacting (phantom) spheres. Many relevant experiments, however, have been performed with polymer chains, which are polydisperse and/or charged and/or self-interacting. These cases are challenging for theoreticians: a limited amount of work performed on these is also discussed here. We particularly concentrate in this review on the direct experimental measurements of the polymer-induced interactions. A brief description of techniques which enable these measurements is given and their strengths and weaknesses are discussed.
\end{abstract}




\section{Contents}

\section{Introduction}

2. Polymer-induced forces; theoretical descriptions

2.1. Non-adsorbing polymers (depletion)

2.1.1.Ideal depletants

2.1.2.Non-ideal depletion cases

2.2. Attached and grafted polymers

2.2.1.Physically adsorbed polymers

2.2.2. Bridging

2.2.3. Grafted polymers

\section{Techniques}

3.1. Surface force apparatus (SFA)

3.2. Atomic force microscopy (AFM)

3.3. Total internal reflection microscopy (TIRM)

3.4. Optical tweezers

4. Analysis of measured forces

4.1. Depletion forces

4.2. Forces induced by attached polymers

4.2.1.Physically adsorbed polymers

4.2.2. Bridging

4.2.3.Grafted polymers

5. Conclusion

6. References

Appendix: List of direct experimental findings on polymer-induced interactions 


\section{Introduction}

It is impossible to imagine people's everyday life without colloidal systems: they are, for instance, ubiquitous in paints, food products, cosmetics, medicines, and biological systems (red blood cells, a living cell, proteins, etc.). One of the key properties for the performance and the storage life of these products is their colloidal stability. This depends on the interactions that are present in the system and how they vary with physical and chemical conditions. There are two different levels at which these interactions can be understood [1]: the first is on a macroscopic level, i. e., collecting knowledge about stability and segregation by observation of macroscopic phenomena. The second one is on a microscopic level, i. e., obtaining the detailed interaction potential between two surfaces as a function of their separation distance by detailed physical experiments. For engineering purposes, the macroscopic level might be sufficient [2-4], whereas for the development of new materials the second, a more detailed, description is necessary [1]. Further, a large amount of theoretical work on colloidal forces and the resulting phase behavior is based on the interaction potential [5]. This creates the need to determine interaction forces or potentials experimentally with sufficient accuracy. Historically, the only way to achieve this goal was to measure structure factors $S(Q)$ of colloidal dispersions by scattering methods, and to calculate the pair correlation function $g(r)$ by Fourier-transformation, which can be related to the pair interaction potential by means of statistical mechanics [6]. This method, however, is susceptible to misinterpretations, since it is, for instance, sensitive to the choice of the closure relation, which is used to calculate the interaction potential from $g(r)$. It is, therefore, desirable to have more direct and model independent methods to measure the force or potential between the colloids. This is now possible using the surface force apparatus (SFA) [7], optical tweezers [8-11], atomic force microscopy (AFM) [12], and total internal reflection microscopy (TIRM) [13, 14].

We review experimental findings obtained using these techniques. A brief introduction of the instruments and a short comparison of their strengths and weaknesses will be discussed here. We concentrate on a description of the progress reached in theoretical and experimental work on polymerinduced pair interactions between dispersed colloids. The effective pair interactions we consider to consist of the direct pair interactions between two colloidal particles plus the interactions mediated by the polymers in the surrounding solution. We disregard many-body interactions between colloids and 
limit the discussion to effective two-body colloidal interactions, because these are directly obtainable from the experimental measurements described here. It is noted that in suspensions where the colloid volume fraction is non-negligible many-body colloidal interactions have to be taken into account to describe the physics of the suspension properly. For example, to calculate the phase behaviour or the structure of colloidal dispersions many-body colloidal interactions are indispensable (e.g. Dijkstra et al. [15]). They are often taken into account by the use of the potential of mean force mediated by other colloids, which however are not necessarily related in a unique way to two-body colloidal interaction potentials [16]. We refrain from the discussion of this issue, as it certainly would deserve a review article on its own. A detailed discussion of various other types of forces and interactions like, van der Waals attraction, electrostatic interactions, structural forces, capillary forces etc. is beyond the scope of this review.

It is known that the presence of a macroscopic surface changes the properties of polymer solutions. For instance, the segment density close to the surface differs from the bulk composition. In the case of adsorption there is an increase of the polymer segment concentration in the surface region. On the other hand, depletion is characterized by a reduction of the polymer concentration close to the surface as compared to the bulk. Whether adsorption or depletion occurs in a system is determined by a very subtle interplay between polymer segment/surface and solvent/surface attractions [17]. If the latter are dominating depletion will occur, while a high affinity of the polymer segments to the interface will favour adsorption. Depletion of polymers from the surfaces of colloidal particles in solution leads to an attractive potential between the particles and, consequently, to a destabilization of the suspension. On the other hand, adsorption of polymers onto colloidal particles may have either a stabilizing or a destabilizing effect. In good solvents (for the polymer) adsorption stabilization, also called steric stabilization, arises and can be attributed to osmotic interactions between segments of the polymers adsorbed onto opposing surfaces. Adsorption flocculation takes place a) due to bridging (if one polymer chain adsorbs onto two or more particles simultaneously) or b) in bad solvents. Thus, the question of polymer induced interactions present in colloidal systems is crucial for their stability. When possible to use, the techniques listed above enable the measurement of interaction potential which, in many cases, provides insight into the colloidal stability. 
In the last 30 years colloidal interactions induced by ideal non-ionic monodisperse polymers were extensively studied using various theoretical methods [17]. This model system enables a detailed theoretical analysis and serves as a starting point for other more complicated systems. However, in many experimental situations the polymers deviate from the assumptions of these theories because they are not ideal, polydisperse or often charged. Another complication can arise when it is not possible to use the Derjaguin approximation [7] to compute the interaction potential (this is true when the size of depletant is comparable or larger than the size of the colloids). All these effects can lead to significant deviations from interactions predicted by the basic theories and are challenging for theoreticians. A limited amount of work performed on such systems is also discussed here.

This review is organized as follows. First, an overview of theoretical achievements is given on forces induced by depleted and attached polymers in section 2 . We describe experimental techniques and compare their strengths and weaknesses in section 3 . In section 4 a survey of directly measured forces and potentials induced by depleted and attached polymers is given, non-ideality of the polymers or colloids in polymer-induced colloidal interactions are discussed as well. The examples discussed in this section were chosen such that they nicely illustrate theoretical predictions. This overview is supplemented by an extensive list of experimental findings, which is given as a table in the appendix [18]. Finally, we give some brief concluding remarks in section 5.

\section{Polymer-induced forces; theoretical descriptions}

Polymer chains in solution have translational, rotational, and conformational degrees of freedom. The presence of the conformational degrees of freedom makes the polymer different from, for instance, colloids and plays an important role in determination of the polymer phase behaviour both in solution and at the surface [19]. In the vicinity of a macroscopic surface the polymer segment density differs from its bulk value. The segment density can be higher than in solution when polymers adsorb onto the surface, or lower in the case if depletion takes place. Whether polymer chains adsorb onto the surface or not is determined by the competition of two factors. First, the fact that the solid surface is impenetrable for the polymer segments causes a reduction of the polymer conformational degrees of 
freedom at the surface. Then, the adsorption behaviour is determined by the effective surface/polymer segment interaction. This can be repulsive or attractive, depending on the solvent, the chemical nature of the polymer and the surface material [19]. All these factors lead to the fact that the adsorption behaviour (adsorption affinity) of a polymer chain is given by a competition between the attractive potential, which tries to bind the polymer segments to the surface, and the entropic repulsion, which tends to maximize entropy, and favours a 'free' state in bulk where a large amount of segments is located far away from the surface.

\subsection{Non-adsorbing polymers (depletion)}

Depletion takes place in solution when the entropic factor dominates; e. g. polymer chains prefer a delocalized state in the bulk. In this case the adsorption affinity of the polymer segments to the surface is low or even repulsive and the gain in potential energy due to surface/polymer segments interactions is lower than the loss due to a reduction of the polymer conformational degrees of freedom at the surface.

The mechanism that is responsible for the resulting depletion interaction can be understood by considering two parallel plates at a distance $h$ immersed in a solution of non-adsorbing non-ionic polymers, as depicted in figure 1. There is a concentration gradient in the average equilibrium polymer-segment concentration profiles when going from the bulk (the maximum segment concentration) to the plate surface (where the concentration is zero). A common simplification to calculate the depletion potential is to replace the concentration profiles with a step function. One part of the step function now consists of a layer in which the polymer concentration equals zero, denoted as a depletion layer with a thickness $\delta$, indicated by the dashed lines along the plate in figure 1 . Outside this layer the polymer concentration equals the bulk polymer concentration. The concentration gradient due to the presence of the depletion layer results in an osmotic pressure gradient. For a single plate this osmotic pressure gradient is balanced. However, if the depletion layers overlap, the osmotic pressure, $\Pi$, becomes unbalanced leading to a net osmotic force that pushes the plates together. In the case of solutions with non-interacting polymers the depletion interaction equals the product of the overlap

volume per unit area, $A, V_{\text {overlap }} / A=2 \delta-h$, (indicated by the hatched area in figure 1) and the 
osmotic pressure, П. Thus, the depletion potential between two parallel plates per unit area can be written as:

$$
\phi_{\text {depl,plates }}(h)= \begin{cases}\infty & \text { for } h<0 \\ -\Pi[2 \delta-h] & \text { for } 0 \leq h \leq 2 \delta \\ 0 & \text { for } h>2 \delta .\end{cases}
$$

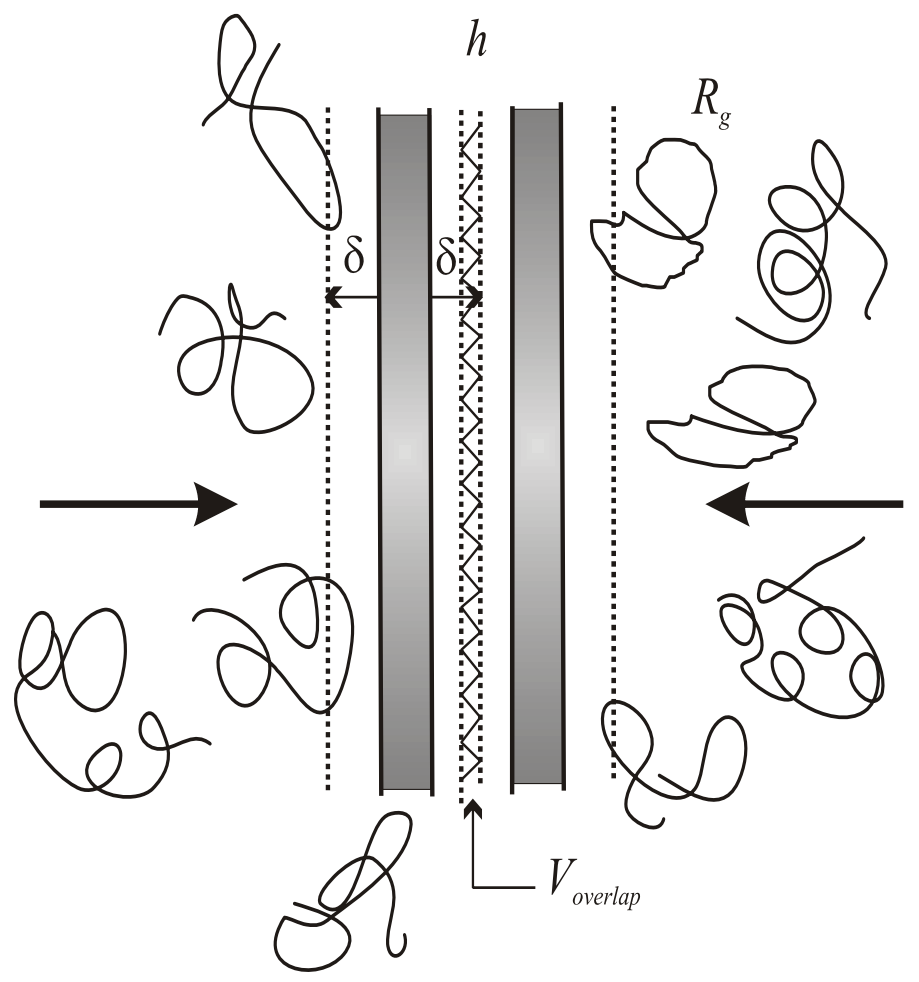

Figure 1. Schematic picture of the depletion zones near two parallel plates in a solution of non-adsorbing polymer molecules. The depletion layers are presented by short dashes. For overlapping depletion layers, shown as hatched area, the osmotic pressure is unbalanced, leading to a net osmotic force, indicated by the arrows, that pushes the plates together.

It is in general easier to derive the interaction potential between two flat plates than between two spheres. However, when the analytical form of the potential is known for plates, one can still compute the interaction potential between two spheres using the Derjaguin approximation [20] if the sphere radii $a_{1}$ and $a_{2}$ are much larger than the range of the interaction [7]:

$$
\phi_{\text {sphere-sphere }}(h)=-2 \pi \frac{a_{1} a_{2}}{a_{1}+a_{2}} \int_{h}^{\infty} \phi_{\text {plate-plate }}\left(h^{\prime}\right) d h^{\prime} .
$$

This directly yields the potential between a sphere and a wall by setting one of the radii $a$ in equation (2) as infinity. 


\subsubsection{Ideal depletants}

The first theory on depletion interaction was published in 1954 by Asakura and Oosawa [21]. They calculated the force between two plates immersed in a solution of non-adsorbing uncharged monodisperse polymers. Using statistical mechanics they derived an expression for the partition coefficient, $\chi$, which is the polymer concentration between the plates divided by the concentration outside the plates. The partition coefficient allows to calculate the osmotic pressure difference between the plates as a function of the separation distance, $h$. Consequently, the interaction potential per unit area between two flat plates, $\phi_{\text {depl }}$, reads

$$
\frac{\phi_{\text {depl,plates }}(h)}{k_{B} T}=-n_{p}[\chi h-h+2 \delta]
$$

where $n_{p}$ is the number density of polymer chains. It is important to note that the knowledge of $\chi$ would generally enable obtaining depletion potentials $[22,23]$. However, $\chi$ can be explicitly calculated only for simple cases.

In the same paper Asakura and Oosawa [21] also calculated the depletion force between two plates immersed in a dilute solution of i) rigid spherical macromolecules, ii) needles (thin rod-like macromolecules) and iii) the force between two spherical bodies with radius $a$ in a dilute solution of rigid spherical macromolecules with radius $R$. In all cases, if $a>>R$, the force is attractive and proportional to the osmotic pressure of the solution, $\Pi$, (which for dilute solutions is given as $\left.\Pi=n_{p} k_{B} T\right)$ and the force range is of the order of the dimension of the macromolecules, $R$. Depletion interaction between two big spheres in a dilute solution of rigid spherical macromolecules can be calculated for $a \gg R$ as [24]

$$
\frac{\phi_{\text {depl,sphere-sphere }}(h)}{k_{B} T}=\left\{\begin{array}{ll}
-2 \pi \cdot n_{p} \cdot a \cdot R^{2}\left(1-\frac{h}{2 R}\right)^{2} & \text { for } 0 \leq h \leq 2 R \\
0 & \text { for } h>2 R
\end{array}\right. \text {. }
$$

Here $n_{p}$ is the number density of polymers. Equation (4) can be obtained also from equation (1), which is valid for the depletion interaction between two flat plates, after applying the Derjaguin approximation (equation (2) 
Simpler than the ideal polymer chain model is the approximation in which the polymers are treated as freely penetrable hard spheres (PHS) whose centres of mass can not approach any non-adsorbing surface closer than a distance of their radius, $R_{P H S}$. PHS are spheres that are hard for a colloidal particle, but which can freely permeate through each other. In 1976 Vrij [25] applied this model to describe colloidal dispersions containing non-adsorbing polymer. If one geometrically calculates the overlap volume between two spheres, $V_{\text {overlap }}$, in a solution of PHS the depletion potential between them can be obtained in a simple analytical form as

$$
\frac{\phi_{\text {depl,sphere-sphere }}(h)}{k_{B} T}= \begin{cases}-\frac{2}{3} \pi n_{p} \cdot R_{P H S}^{3}\left(1-\frac{h}{2 R_{P H S}}\right)^{2}\left(2+\frac{3 a}{R_{P H S}}+\frac{h}{2 R_{P H S}}\right) & \text { for } 0 \leq h \leq 2 R_{P H S} . \\ 0 & \text { for } h \geq 2 R_{P H S}\end{cases}
$$

where $n_{p}$ is the number density of polymers. This expression is equivalent to the equation for the depletion potential between two big spheres mediated by a dilute suspension of small spheres given by Asakura and Oosawa [24], if $R_{P H S}$ is replaced by the radius of the small spheres. For the case $R_{P H S}<<$ $a$, and $h<<a$, equation (5) reduces to equation (4).

Classically, in equation (5) the radius of gyration of polymer, $R_{g}$, was taken for the radius of the PHS, $R_{P H S}$, as was also done by Vrij [25]. However, it should be the depletion thickness $\delta$. De Hek and Vrij [26] discussed this issue as well in the appendix of their paper. To determine $\delta$ one has to compute the segment density profile of ideal chains near a single wall, that was done by Eisenriegler [27]. The integration of this profile provides $\delta[23]$ :

$$
\delta=\frac{2 R_{g}}{\sqrt{\pi}},
$$

which is close to the radius of gyration of the polymer, $R_{g}$.

The PHS approximation is a very good model for ideal chains to describe the interactions between flat walls and for large spheres. Significant deviations appear for $R_{P H S}>a$. The validity of the PHS model for polymers is extensively discussed in the review of Tuinier et al. [28].

\subsubsection{Non-ideal depletion cases}


Since polymer molecules are not spheres, but rather fluctuating objects, they will not be completely excluded from the region between flat walls or colloidal particles, as it is assumed within the PHS model. More precise descriptions take the statistical properties of polymers into account enabling a more accurate prediction of the concentration of the polymer segments in the depletion zone [29-34]. Furthermore, in many classical descriptions polymers were assumed to be ideal [21, 25]. In reality, polymer chains interact due to excluded volumes of their segments, which can be (partly) compensated due to possible attraction between segments mediated by the solvent quality. Mean-field (MF) and scaling theories enable including excluded volume interactions and the statistical properties of the polymer molecules. Scaling theories for depletion interaction were first proposed by de Gennes [35] and Joanny et al. [33]. MF theories by Feigin and Napper [32], and Scheutjens and Fleer [34, 36] qualitatively differ from those of Asakura and Oosawa, and de Gennes with co-workers in the sense that they predict not only depletion attraction but also depletion stabilization. It should be mentioned that the term stabilization in this case does not imply a repulsive barrier in the interaction potential but rather a weakening of the attractive depletion effect. This is due to the depletion thickness following the polymer correlation length, which vanishes at high concentrations. An experimental verification of this effect was found by Cowell et al. [37]. The depletion stabilization was found above the semidilute concentration regime, where scaling arguments could not support this.

De Gennes [38] considered the contact potential between two colloidal spheres in a semi-dilute polymer solution in a good solvent for very large spheres, where the only relevant length scales are the sphere radius $a$ and the correlation length $\xi$, leading to the following scaling relation for the minimum of the interaction potential [38]:

$$
\frac{\phi_{\text {depl }, \text { sphere-sphere }}(h=0)}{k_{B} T}=-\lambda \cdot \frac{a}{\xi} \text {. }
$$

For the situation $a>>\xi$ the depletion attraction between the two spheres is predicted to be very strong. In the excluded volume regime the pre-factor $\lambda$ in equation (7) was calculated by Tuinier et al. [39] and equals $\lambda=0.45$.

Joanny et al. [33] derived an expression for the effective depletion interaction where the polymer excluded volume effect was taken into account. The authors used the elementary MF theory and a 
scaling approach. To predict the interaction potential between two big spheres as a function of the polymer concentration and chain length in semi-dilute polymer solutions the scaling theory was applied. It was shown that the range of the interaction scales with the polymer correlation length, $\xi$, and decreases with increasing polymer concentration in the semi-dilute regime according to the following equation:

$$
\frac{\phi_{\text {depl,sphere-sphere }}}{k_{B} T} \sim\left\{\begin{array}{ll}
-\frac{a}{\xi^{3}}(\pi \xi-h)^{2} & \text { for } 0 \leq h \leq \pi \xi \\
0 & \text { for } h>\pi \xi
\end{array} .\right.
$$

As the correlation length $\xi$ scales with the polymer mass concentration $c$ as $\xi \sim c^{-3 / 4}[35]$ the range of the potential decreases with increasing polymer concentration.

Feigin and Napper [32] calculated the free energy of interaction between two flat plates as follows from the conformation of polymers between two particles. They found a repulsive part in the depletion interaction between the plates in the concentrated polymer regime. The contact potential, however, was still negative.

Scheutjens and Fleer introduced a numerical lattice self-consistent field (SCF) method and computed the interaction potential between two flat walls in a highly concentrated polymer solution ( $>10 \%$ volume fraction of polymer segments) [34]. At this condition a weak repulsive part was found in the potential. However, in the case of polymer chains in a theta solvent the depletion interaction was shown to be only attractive. The disadvantage of SCF lattice computations is that they only give numerical results; so, this method does not provide an analytical expression for the depletion interaction. However, it enables investigating trends in complicated systems. Scaling methods apply for weakly overlapping, long flexible polymer chains in semi-dilute concentration regime, where MF approaches produce incorrect results. The Scheutjens-Fleer theory, however, can be extended to a variety of systems, such as polydisperse samples, mixtures of different polymers, block-copolymers, polyelectrolytes [40], etc.

Tuinier and Fleer [22] presented a simple analytical MF theory for the pair interaction between two colloidal particles, based upon a MF equation [41] for the depletion thickness $\delta$ which depends on the polymer chain length, the bulk concentration, and the solvent quality. Only in the extremely dilute case 
(in MF) the interaction was found to be independent of the solvent quality. At relevant concentrations a better solvency leads for two flat plates to a stronger attraction at contact and a smaller range of attraction. In the dilute limit $\delta$ equals to the depletion thickness of ideal chains. For the interaction between two spheres of radius $a$ the contact potential is nearly insensitive to solvency; again, the range of attraction is smaller for better solvents.

Further, Tuinier et al. [42] studied the interactions between two colloids in a solution based on the generalized Gibbs adsorption equation, which allows to calculate the interaction potential from the amount of polymer adsorbed onto the particle interface [43]. Starting from appropriate expressions for the correlation length and the osmotic pressure of a polymer solution in the excluded volume interaction limit, the authors computed the interaction potential between two plates, two spheres and between a sphere and a plate. The results were found to be in agreement with computer simulation results of Bolhuis et al. [44]. Figure 2 presents the interaction potential obtained by computer simulations of self-avoiding random walks (SAW) in a good solvent between two parallel flat plates performed by Bolhuis et al. [44]. Here the interaction potentials for three polymer concentrations are plotted as a function of the relative plate separation distance, $h / R_{g}$. The values of the contact potentials of the SAW simulations (symbols) and their range correspond reasonably well to the theoretical predictions (full curves) of Tuinier et al. [42].

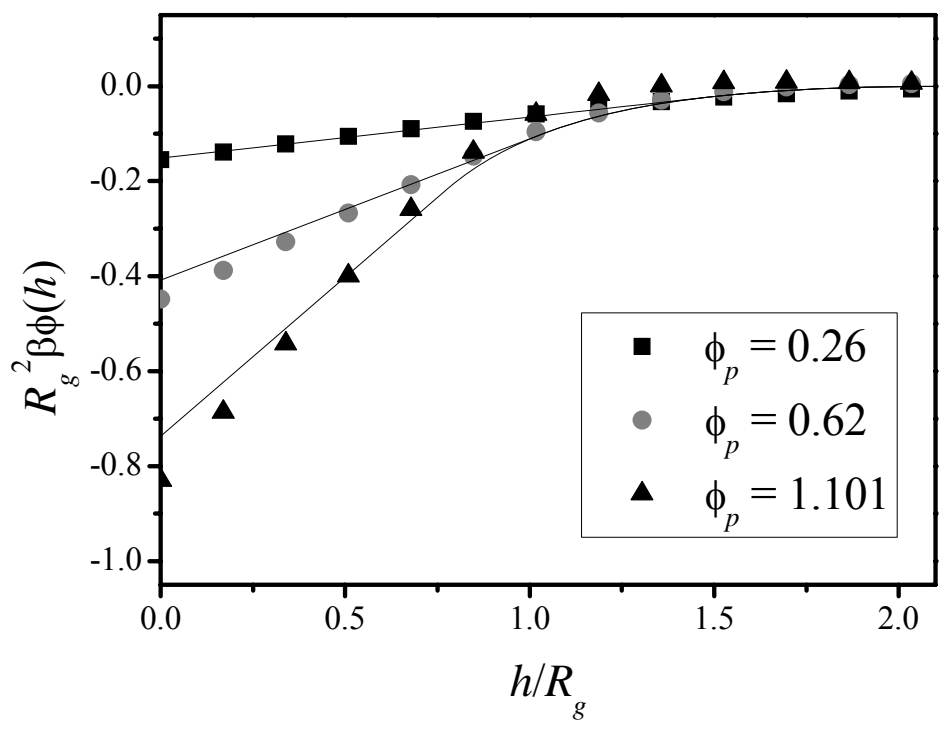


Figure 2. Interaction potential between two hard walls immersed in a solution with selfavoiding walk polymers as a function of the distance between the plates for different polymer concentrations, as indicated. The symbols refer to simulations results from [44] and the full curves are the prediction of the theory for interacting polymers from Tuinier et al. [42].

Recently, Pelissetto and Hansen [45] obtained effective pair interaction potentials between hardsphere colloids and the centers of mass of self-avoiding polymer coils in the low-density limit by Monte Carlo (MC) sampling of polymer conformations, over a wide range of size ratio $a / R_{g}$. Simulations carried out for several polymer molar masses allowed the authors to extrapolate the data to the scaling limit (where the length of the polymer chain is infinitely long $R_{g}=\infty$ ).

Also density functional theories were used to describe depletion interactions. Thus, the potential between two big spheres in a solution of interacting small spheres was calculated by Roth et al. [46]. A modified version of this approach was successfully applied to describe the interaction potential between a sphere and a wall in a non-adsorbing polymer solution as well as mediated by a solution of rods, as measured by TIRM $[47,48]$.

Charged polymers. In many polymer-colloid mixtures, especially in aqueous solutions, charges are present either on the polymer chains or on the particles or on both of them. In their 1958 paper Asakura and Oosawa extended their depletion theories and treated the cases of interaction in solutions of charged macromolecules [24]. They showed that with the appearance of charges on polymers, both the range of the interaction and the absolute value of the potential energy increase. Expressions were given to estimate the force, $f_{\text {depl,plates }}(h)$, and the potential energy $\phi_{\text {depl,plates }}(h)$ between two neutral plates in a solution of charged macromolecules.

Böhmer et al. [40] numerically computed depletion interaction due to polyelectrolytes using the Scheutjens-Fleer lattice approach. For polyelectrolytes the interaction was found to be mediated by the salt concentration in the system.

Ferreira et al. [49] studied the equilibrium and structural properties of mixtures containing nonadsorbing flexible polyelectrolytes and like-charged colloids using liquid state integral equations. The polymer chains in the semi-dilute regime were treated within the polymer reference interaction site 
model. The monomers and the colloids assumed to interact via screened Coulomb potentials. The electrostatic interactions between the different macromolecules inside the solution were found to be responsible for complex local structures and rich phase diagrams, which depended on the number of charges and on the Debye screening length.

Soft surfaces. The theories mentioned so far were restricted to the polymer-mediated interactions between hard surfaces. Experimentally, 'soft' surfaces are often used, e.g., when the particles are surrounded by a layer of grafted polymer hairs. In this case, the definition of the depletion thickness is more complicated because some interpenetration and/or compression of the hairs by the non-adsorbing polymer chains may occur [50]. This effect can lead to crucial deviations from the classical predictions of the depletion force (see section 2.1.1. Ideal depletants).

Feigin and Napper [32] used the rotational isomeric state-depletion MC procedures and the FloryHuggins MF theory to show that the presence of additional attached polymer chains at the surface may have a significant effect on depletion interaction due to 'free' chains between two particles. Such a steric layer tends to counteract the depletion interaction, thereby increasing the concentration of free polymer needed to induce depletion flocculation. The effect of the steric chains, however, is reduced if the free polymer are much longer than the grafted chains [51].

Striolo [52] used MC simulations to calculate the effective interactions between colloidal brushes dissolved in a solution of non-adsorbing polymer. A weak mid-ranged attraction was observed and attributed to depletion for surfaces with brushes consisting of 5 segments in a solution of nonadsorbing polymer chains with 10 segments. However, the strength of the induced attraction was weaker than the depletion attraction computed for HS colloids in solutions of non-adsorbing polymers. It was also shown that, under the conditions Striolo chose, it is not possible to superimpose depletion attraction and steric repulsion to predict the correct interaction potential between the surfaces bearing brushes in solutions of non-adsorbing polymer chains.

MC simulations were performed by Broukhno et al. [53] to study a colloidal dispersion whose stability was manipulated by adding adsorbing and/or non-adsorbing polymers. An attractive depletion force was found for non-adsorbing polymer chains. At high volume fractions of depletants the 
attraction turned into a repulsion at short separation distances. Depletion was also found in the case of an intermediate adsorption affinity (see section 2. Polymer-induced forces; theoretical results), while strong adsorption gives rise to a significant attraction through bridging.

Polydisperse polymers. An essential issue that has not attracted much attention in theories and simulations is polydispersity. Because of the characteristic kinetics of polymerization, most synthetic and natural (except for several proteins and viruses) polymers have a finite molar mass distribution. However, polymers are often treated as being monodisperse and incorporation of the size polydispersity of polymers has gained very limited attention in theories for (polymer-induced) depletion. So far, polydisperse polymers were mainly simplified as polydisperse spheres [54-60]. A first extension towards polydisperse ideal chains as depletants was made by Tuinier and Petukhov [61]. For the depletion interaction between two plates due to ideal polydisperse polymer chains they derived an exact expression:

$$
\frac{\phi_{\text {depl }, \text { plate-plate }}}{k_{B} T}=-n_{p}\left(\chi_{\text {poly }} h-h+2 \delta_{\text {poly }}\right) \text {. }
$$

Equation (9) has a mathematical form similar to equation (3) from Asakura and Oosawa, but with different, polydispersity-dependent functions $\chi_{\text {poly }}$ and $\delta_{\text {poly }}$, which are functions of the polymer molar mass distribution. The MC simulations performed by Tuinier and Petukhov [61] showed that confinement of depleted chains leads to a size fractionation of the polymers; larger chains tend to be more expelled from a narrow slit than small chains. Later, Kleshchanok et al. [62] gave an expression for the depletion interaction between a plate and a sphere in the solution of ideal polydisperse polymers using the Derjaguin approximation.

It is important to note that the effect of polydispersity on the depth and the contact value, $\phi(h=0) / k_{\mathrm{B}} T$, of the depletion potentials appears to depend on the measure for the polymer content, which is kept constant when comparing monodisperse to polydisperse depletants. Piech and Walz showed that the absolute value of $\phi(h=0) / k_{\mathrm{B}} T$ caused by spherical depletants is smaller for polydisperse depletants than for monodisperse spheres at constant volume fraction, $\eta$. On the other hand, at constant number density, $n_{\mathrm{p}}$, polydisperse spheres cause a deeper depletion potential than 
monodisperse ones [57]. This effect is caused by the fact that different measures for the content of depletants are related by the distribution functions of the polymer size and the polymer mass by

$\eta_{\text {poly }}=n_{\text {poly }} \frac{\int_{0}^{\infty} V_{p} \Psi\left(V_{p}\right) d V_{p}}{\int_{0}^{\infty} \Psi\left(V_{p}\right) d V_{p}}$

with

$n_{\text {poly }}=c N_{A} \frac{\int_{0}^{\infty} \frac{1}{M} \Psi(M) d M}{\int_{0}^{\infty} \Psi(M) d M}$

where $V_{\mathrm{p}}$ is the single depletant volume $c$ the polymer mass concentration and $M$ the molar mass. If $\Psi$ is the number fraction of either quantity, the two distribution functions are the same, $\Psi(M)=\Psi\left(V_{p}\right)$, and we may express equation (10) in terms of the typical length, $l$, of the depletant as

$\eta_{\text {poly }}=n_{\text {poly }} X \frac{\int_{0}^{\infty} l^{3} \Psi(l) d l}{\int_{0}^{\infty} \Psi(l) d l}$

with

$n_{\text {poly }}=\frac{c N_{A}}{Y} \frac{\int_{0}^{\infty} \frac{1}{l} \Psi(l) d l}{\int_{0}^{\infty} l^{\mu-1} \Psi(l) d l}$

taking into account that for any particle geometry $V_{\mathrm{p}}=X l^{3}$ and $M=Y l^{\mu}$. As an example we discuss the case of spherical depletants in more detail. There $l$ corresponds to the radius $R_{\mathrm{PHS}}, X=4 \pi / 3, Y=X d_{\mathrm{p}} N_{\mathrm{A}}$ and $\mu=3$. Thus we get

$\eta_{\text {poly }}=n_{\text {poly }} \frac{4 \pi}{3} \frac{\int_{0}^{\infty} R_{P H S}^{3} \Psi\left(R_{P H S}\right) d R_{P H S}}{\int_{0}^{\infty} \Psi\left(R_{P H S}\right) d R_{P H S}}$

with

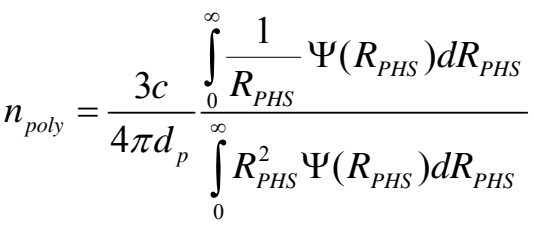


with $d_{\mathrm{p}}$ the particle mass density. To illustrate the effect of the different weighting in the three measures for the depletant content, we show calculated depletion potentials between a sphere of radius $a=3 \mu \mathrm{m}$ and a flat wall mediated by a solution of spherical depletants with a mean radius of $\left\langle R_{H P S}\right\rangle=$ $100 \mathrm{~nm}$ and $d_{\mathrm{p}}=1 \mathrm{~g} / \mathrm{L}$ in figure 3 . For the case of monodisperse depletants we used $c=100 \mathrm{~g} / \mathrm{L}$ corresponding to $\eta=0.1$ and $n_{\mathrm{b}}=190 \mu \mathrm{m}^{-3}$. At constant volume fraction and a relative standard deviation $\sigma=0.5$ (log-norm distribution) equation (12) yields $c=47 \mathrm{~g} / \mathrm{L}$ and $n_{\text {poly }}=62 \mu \mathrm{m}^{-3}$. The latter value explains why the absolute contact value of the potential calculated with these parameters is smaller than in the case of the monodisperse depletants. Differently, if the concentration is kept constant, polydispersity will cause an increase of the number density, which causes a deepening of the interaction potential. As expected, the range of the potential increases in all cases.

For ideal chains or rod-like polymers as depletants the same formalism can by applied with differing results. In the case of ideal chains, polydisperse depletants cause a smaller absolute contact value than monodisperse polymers at the same volume fraction. The potentials obtained at constant concentration and at constant density coincide and are deeper than that mediated by monodisperse depletants. For rod-like depletants, the potentials caused by polydisperse polymers always have a larger absolute contact value than the potential mediated by monodisperse rods. 


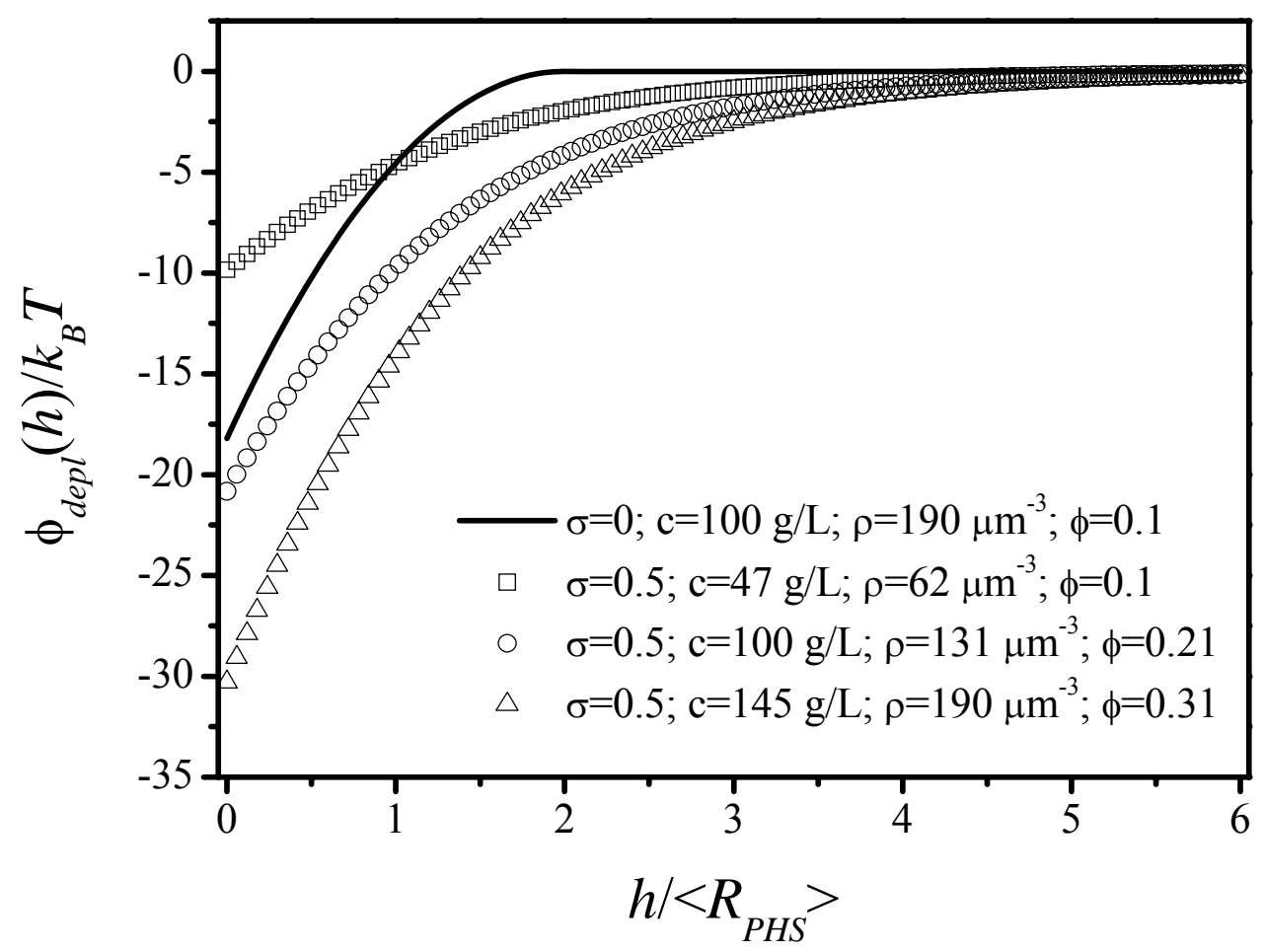

Figure 3. Depletion between a flat wall and a sphere with $a=3 \mu \mathrm{m}$ mediated by spherical polymers of mean number-averaged radius $\left\langle R_{P H S}>=100 \mathrm{~nm}\right.$. The full line corresponds to a monodisperse depletant with $c=100 \mathrm{~g} / \mathrm{L}$ corresponding to $\eta=0.1$ and $n_{\mathrm{b}}=190 \mu \mathrm{m}^{-3}$. The symbols correspond to polydisperse depletants with $\sigma=0.5$ at the same volume fraction (squares), the same concentration (circles) or the same density (triangles) as the monodisperse polymer.

The effect of the exact shape of the polymer size distribution on the depletion potential between a sphere $(a=3 \mu \mathrm{m})$ and a wall in solution with a polymer concentration of $0.1 \mathrm{~g} / \mathrm{L}$ is demonstrated in figure 4 for the case of ideal chains as depletants. The polymer radii of gyration $R_{g}$ are assumed to follow the Schulz-Zimm (SZ) and the log-normal (LN) distributions [63], with a mean radius of gyration $R_{g}$ of $100 \mathrm{~nm}$. The distributions are shown as an inset in figure 4 with the width of the $\mathrm{SZ}$ distribution $z=3$ which is related to the relative standard deviation by $\sigma=1 / \sqrt{z+1}$. The relative standard deviation of the LN distribution was set at $\sigma=0.5$. The major differences between the 
potentials calculated with different distribution functions are a larger absolute contact value and a shorter range of the potentials mediated by SZ distributed polymers. This can be qualitatively understood from the shape of the distributions. One can see that the fraction of polymer chains with smaller $R_{g}$ is larger in the case of the $\mathrm{SZ}$ distribution. This means that the depletion interaction due to this kind of polymers will be shorter-ranged. On the other hand, the contact potential will increase because of increased polymer number density. However, the differences in the depletion potential caused by the type of the polymer size distribution are pronounced for distributions with $\sigma>0.5$.

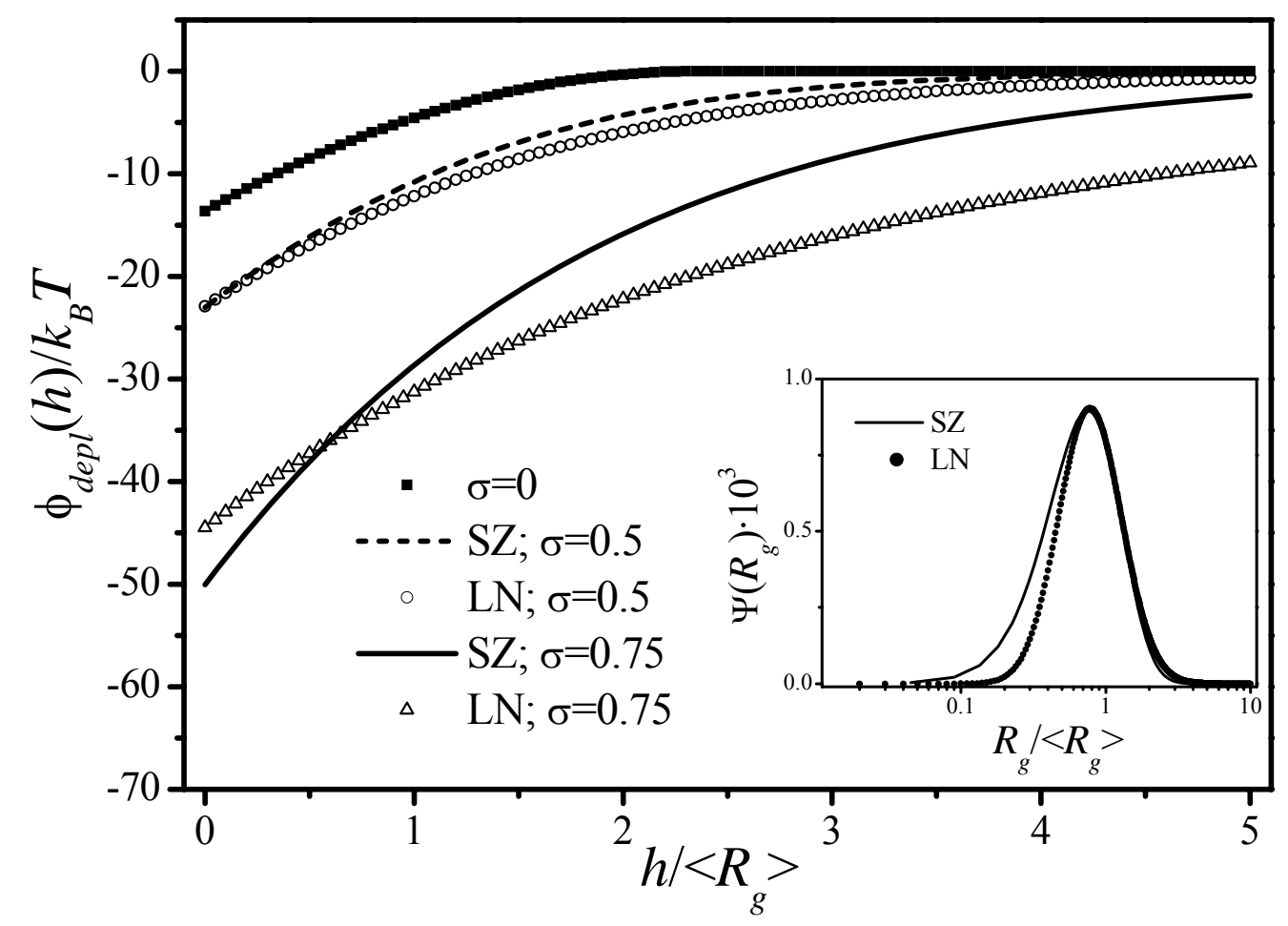

Figure 4. Calculated depletion interaction potentials, $\Delta \phi_{\text {depl }}(h)$, between a sphere $(a=3 \mu \mathrm{m})$ and a wall in solution of polydisperse polymers with various $R_{g}$ distributions: $\mathrm{LN}$ and $\mathrm{SZ}$ with parameters indicated in the legend. Inset: distributions of polymer radius of gyration with $\sigma=0.5$. The mean radius of gyration for all cases was $<R_{g}>=100 \mathrm{~nm}, c_{p}=0.1 \mathrm{~g} / \mathrm{L}$ 
Superposition approximation. When several types of interactions take place between two bodies in a system, the total potential can be estimated using the superposition approximation:

$$
\phi_{\text {tot }}(h)=\sum_{i} \phi_{i}(h)
$$

This procedure is successfully used, for instance, for analyzing the potentials obtained with TIRM [13]. Figure 5 presents the calculated total interaction potential between a charged $5.7 \mu \mathrm{m}$ diameter particle and a charged wall at the various Debye lengths and polymer concentrations $\left(R_{g}=44 \mathrm{~nm}\right)$ in the solution. The curves were calculated by summation of electrostatic repulsion, gravity and depletion.

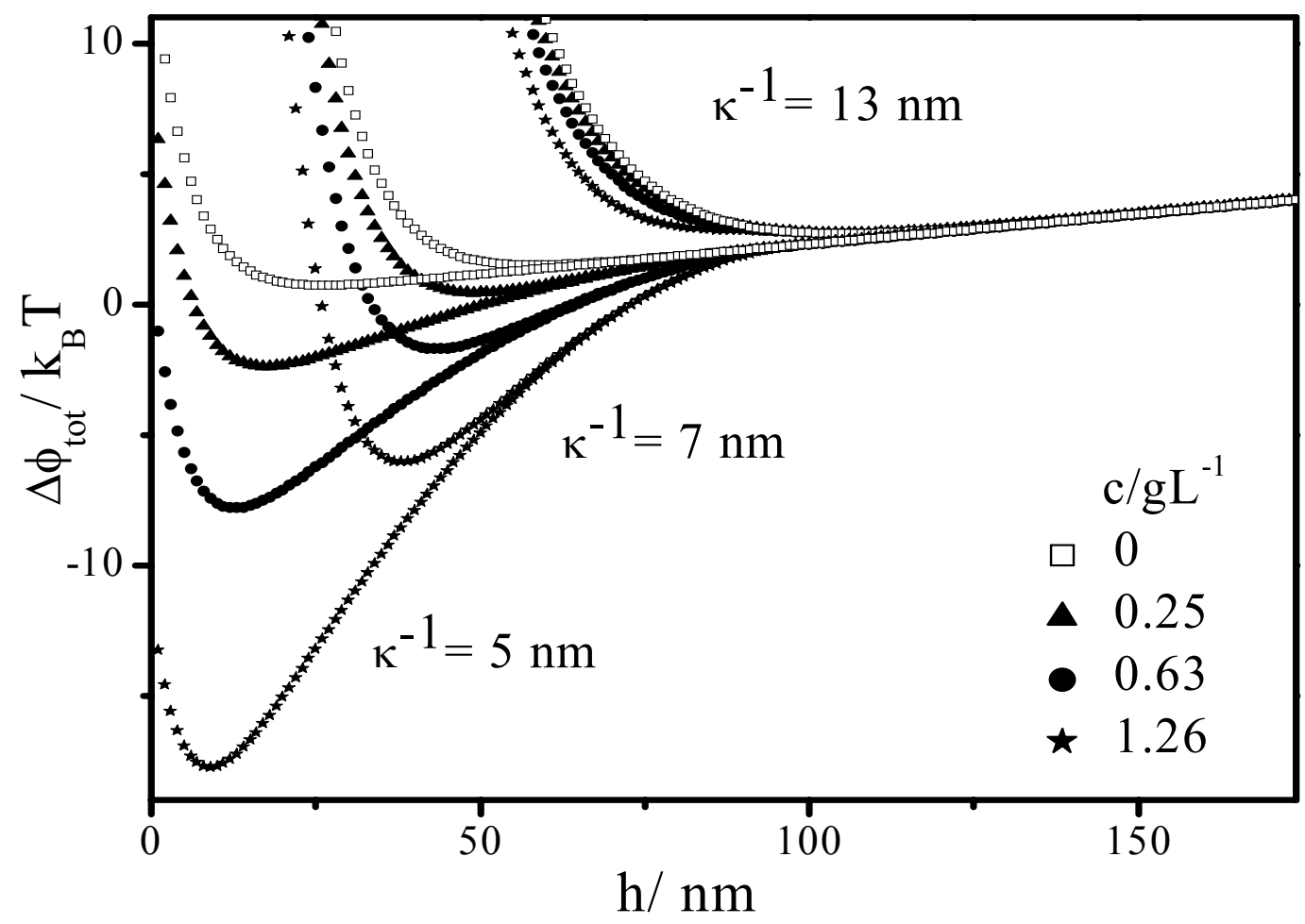

Figure 5. Calculated total interaction potentials, $\Delta \phi_{\text {tot }}(h)$, between a charged $5.7 \mu \mathrm{m}$ diameter particle and a charged wall in a solution of polymer with the $R_{g}=44 \mathrm{~nm}$ at the various Debye lengths. The polymer concentrations of the solutions are indicated in the legend.

In contrast, Croze and Cates [64] predict, on the basis of MF theory, that non-ionic polymers confined between charged flat surfaces couple electrostatic and depletion effects in a non-additive way. The authors found that the depletion effects were enhanced electrostatically and that electrostatic 
interaction was effectively screened. This finding was explained by polarization effects due to the low dielectric permittivity of polymer segments with respect to a high-permittivity solvent (e.g., water).

Depletion between non-spherical colloids. In 1958 Asakura and Oosawa considered the case of interaction in solutions of asymmetrical macromolecules, which they described as rigid ellipsoids [24]. They showed that an increase in dissymmetry of solute macromolecules causes an increase in both the range and the strength of the interaction potential. Exact expressions for the depletion interaction mediated by rod-like particles between two plates and two big spheres were derived in 1981 by Auvray [65] and later by Mao et al. [66, 67], also for the high concentration regime of rods. In their theory the length $L$ of the rod-like particles with a diameter $D$ is much smaller than the radius $a$ of the colloidal spheres. To the lowest order in rod density the depletion potential is given by [67]:

$$
\frac{\phi_{\text {depl }, \text { sphere-sphere }}(h)}{k_{B} T}= \begin{cases}-\frac{\pi}{6} n_{R} \cdot a \cdot L^{2}\left(1-\frac{h}{L}\right)^{3} & \text { for } 0 \leq h \leq L \\ 0 & \text { for } h>L\end{cases}
$$

where $n_{R}$ is the number density of the rods.

The depletion interaction between ellipsoidal colloidal particles in a solution of long ideal polymers were analyzed by Eisenriegler [68]. Special attention was given to the limiting cases in which the ellipsoid reduces to a cylinder of infinite length and finite radius and a 'needle' of finite length and vanishing radius. Exact quantitative results were obtained for the orientation-dependent depletion interaction between a short needle and a wall.

De Vries [69] derived simple analytical approximations for the depletion attraction between rod-like segments of semi-flexible polyelectrolytes such as DNA, induced by non-adsorbing globular proteins. His approach is based on a virial expansion of the polyelectrolyte chemical potential. MC simulations [69] show that the approximation is accurate up to protein volume fractions of at least $20 \%$. It was found that non-adsorbing globular proteins are much less efficient depletants than inert flexible polymers.

Yaman et al. [70] computed numerically the depletion potential between two spheres in a solution of rods with the length $L$ for all size ratios $L / a$ and, indeed, for $L / a>0.1$ they found deviations from the values obtained with the Derjaguin approximations, the more so for larger values of $L / a$. 


\subsection{Attached polymers}

Polymer layers at the surfaces can be created in three different ways: (i) firstly, due to physical adsorption; (ii) secondly, polymers can be chemically grafted to the surface and (iii) thirdly, in the case of, for instance, diblock copolymers they can be anchored by an insoluble part [19]. A typical configuration of an adsorbed polymer at a surface is sketched in figure 6. 'Trains' are polymer parts which are bound to the substrate and are in direct contact with it. Between trains one finds chain sections that are not in direct contact with the surface denoted as 'loops' and the dangling ends of the chains are called 'tails'. These terms were proposed by Jenkel and Rumbach [71].

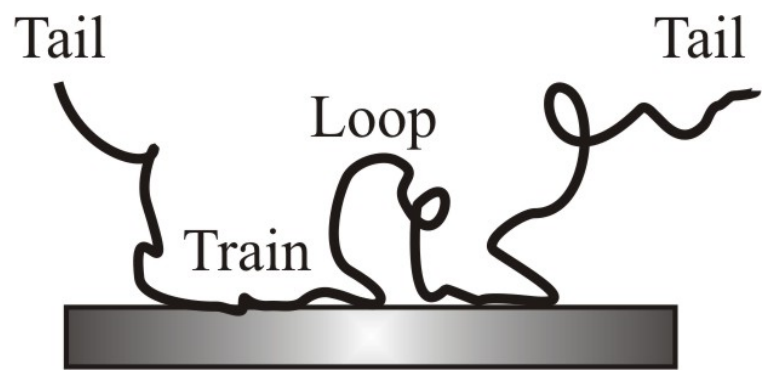

Figure 6. Polymer chain adsorbed at the surface consisting of tails, loops and trains.

If the polymers are grafted or anchored to the surface their chains can assume three different structures depending on grafting density, as it shown in figure 7. When the distance between isolated chains is larger than the order of the radius of gyration $R_{g}$, two limiting cases can be found depending on the adsorption affinity of the polymer segments: a) a mushroom in case of non-adsorbing segments and b) a pancake in case of adsorbing segments. In case of denser polymer layers the chains become stretched forming brushes (figure 7c). These names were first proposed by de Gennes [72].

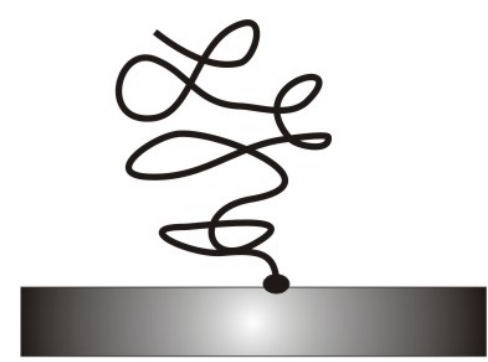

a) mushroom

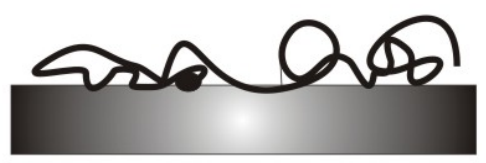

b) pancake

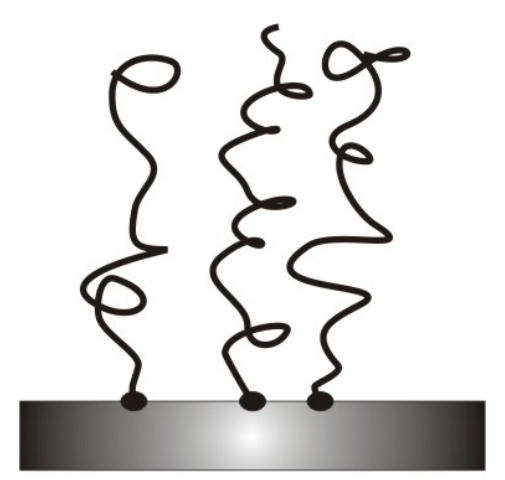

c) brush

Figure 7. Schematic picture of three limiting structures of grafted or anchored polymer chains. 
Theoretically, polymer adsorption and the interactions between polymer-covered surfaces are often examined using either scaling or MF theories or via computer simulations $[7,17,73]$. Polymer adsorption leads to either stabilization or flocculation, depending on a number of factors, such as: the amount of polymer attached to the surface, solvent quality and whether the polymer is chemically or physically attached to the surface. Adsorption stabilization, also called steric stabilization, arises in a good solvent and can be attributed to the osmotic interactions between the polymer segments on opposite surfaces. Adsorption flocculation occurs either due to bridging, when polymer chains adsorbs on several surfaces simultaneously when there is not enough polymer to fully cover the surfaces, or due to bad solvent conditions for the adsorbed polymer layers.

\subsubsection{Physically adsorbed polymers}

Theories of steric interactions are complex and different approaches can be distinguished in literature $[7,74]$. If the surfaces are covered with adsorbed polymer chains under the condition of full equilibrium with the surrounding solution, the theoretically predicted forces between two polymer adsorption layers are attractive arising from bridging [75, 76]. However, it appears that under experimental conditions full equilibrium is not reached in dense adsorbed polymer layers [19]. Weak repulsion was also theoretically found at larger separation distances [77], which, however, is not always strong enough to withstand the van der Waals attraction between the colloidal particles and stabilize them [78].

In the so-called case of constrained equilibrium the total number of chains between two surfaces is fixed. Under these conditions MF computations show that the polymer coated surfaces attract one another if the surfaces are not saturated, while the force is repulsive at full coverage. Further, the situation also depends on the solvent quality. De Gennes predicted a repulsive interaction for strongly overlapping chains in good solvents. The repulsion arises from the steric interaction between two opposing adsorption layers [75]. In a bad solvent, in addition to a long-ranged attraction a short-ranged repulsion is found due to compression of the adsorbed polymer layers $[76,79,80]$. The attractive interaction occurs because the polymer concentrations in the interfacial region lie in the unstable twophase region of the bulk phase diagram. The case of constrained equilibrium is relevant, for example, 
for measurements performed with the surface force apparatus (SFA) [80-83] (see section 3.1. Surface force apparatus (SFA)).

\subsubsection{Bridging}

Strong attraction at large separation distances due to bridging takes place between two undersaturated polymer layers. This kind of attraction is responsible for coagulation in colloidal systems, when the amount of added polymer is not high enough to fully cover the total surface of the colloids [84]. There are analytical theories available nowadays to describe this bridging effect. Bhatia and Russel [85] used the Dolan-Edwards approach [86] to treat telechelic polymers (ABA type) as ideal chains. Analytical expressions for flat plates and numerical results for chains between two spheres were obtained. For flat plates, the attraction of $0.6 k_{B} T$ per chain occurs at a separation of roughly one end-to-end distance and is stronger than for good solvents. Some modifications in the model of Bahatia and Russel were made by Porte et al. [87]. They accounted for the fact that at finite droplet volume fraction the polymer-induced bridging interaction between two droplets in the microemulsion should be calculated at constant chemical potential of the polymer chains rather than at fixed grafting density.

Cao and $\mathrm{Wu}[88]$ investigated the telechelic polymer-mediated surface forces by using a polymer density functional theory (PDFT). Within a single theoretical framework, the PDFT is able to capture both the depletion induced attraction in the presence of polymers which have a weak adsorption affinity and the steric repulsion between compressed polymer brushes. The authors found that the weak attraction between surfaces covered with telechelic chains is primarily caused by bridging.

\subsubsection{Grafted polymers}

If polymer chains are end-grafted onto the surface with sufficiently high grafting density they act as very efficient stabilizers for colloidal particles in the good solvent regime. The interaction between two surfaces bearing grafted polymers in full equilibrium is repulsive [89-91] as bridging does not take place between such surfaces. 
The interaction between particles with a radius $a$ bearing polymer brushes with the brush height $H_{\text {brush }}$ can be described by the simple Alexander-de Gennes model for polymeric brushes $[89,90]$ :

$$
\begin{aligned}
& \frac{\phi_{\text {brush,sphere-sphere }}(h)}{k_{B} T}= \\
& = \begin{cases}\infty & \text { for } h<0 \\
\frac{16 \pi a H_{\text {brush }}^{2} \sigma_{\text {brush }}^{\frac{3}{2}}}{35}\left[28\left(\left(\frac{2 H_{\text {brush }}}{h}\right)^{\frac{1}{4}}-1\right)+\frac{20}{11}\left(1-\left(\frac{h}{2 H_{\text {brush }}}\right)^{\frac{11}{4}}\right)+12\left(\frac{h}{2 H_{\text {brush }}}-1\right)\right] \text { for } 0 \leq h \leq 2 H_{\text {brush }} \\
0 \quad \text { for } h>2 H_{\text {brush }}\end{cases}
\end{aligned}
$$

Here $\sigma_{\text {brush }}$ is the grafting density expressed as (a) the number of brush chains per unit area. The Alexander-de Gennes approach derives from a scaling theory which assumes a step-like segment density profile with all chains ending at the edge of the brush. MC simulations and numerical MF calculations show that the brush height exhibit a more parabolic monomer density profile which goes to zero in a continuous manner at the outer perimeter [92]. Nevertheless, a more advance MF treatment [93] predicts a very similar force law to the Alexander-de Gennes equation (equation (15)).

Manciu et al. [94] suggested a simple model to calculate the monomer density and the interaction between surfaces with grafted polymer brushes, based on an approximate MC calculation of the partition function of the polymer chains. This model could be employed for both good and poor solvents. It was found to be compatible with a parabolic-like profile at moderate grafting densities, and leads to an almost step-like density for highly stretched brushes. In good and moderately poor solvents, the interactions between surfaces with grafted polymer brushes were found to be repulsive always, whereas in poor solvents the interactions were repulsive at small separations and became attractive at intermediate separation distances.

Charged brushes. Miklavic and Marcelja [95] and Misra et al. [96] extended the analytical SCF theory of grafted polymer brushes introduced by Milner et al. [93] to calculate the interaction between two polyelectrolyte chain layers attached to charged surfaces. Pincus [97] studied the structure of a dense polyelectrolyte layer grafted to the surface. He suggested a simple theory for the scaling properties of the layer thickness, counterion distribution and disjoining pressure between two opposing layers. The flat layers studies were extended to the case of colloidal spheres. Thus, two regimes have 
to be distinguished for charged brushes: an "osmotic brush" and a "salted brush". For an "osmotic brush" nearly all counterions are located inside the brush due to strong electrostatic interaction of the polymers. The force for stretching the chains perpendicularly to the surface is proportional to the osmotic pressure of counterions multiplied by surface area per chain. The brush thickness $H_{\text {brush }}^{o s m}$ in this regime results from the equilibrium between this osmotic force and the oppositely directed elastic force [97]

$$
H_{\text {brush }}^{\text {osm }} \approx N m \alpha^{0.5}
$$

where $N$ is the polymerization degree, $m$ is the monomer size, and $\alpha$ is the fraction of charged monomers in the chain. The equation (16) is valid except for weakly charged polymers and low grafting densities. In the osmotic brush regime the thickness is independent of the grafting density in the scaling approximation if non-power dependencies are neglected. For a "salted brush" an external salt concentration $c_{\text {salt }}$ of ions is present in the solution in addition to the charges of the polyelectrolyte brushes. It results in screening of the charges along the chain. Therefore the osmotic pressure on the chain is reduced. If the concentration of the added salt exceeds the concentration of counterions inside the brush, the equilibrium brush thickness is given by [97, 98]

$$
H_{\text {brush }}^{\text {salt }} \approx N \sigma_{\text {brush }}^{1 / 3} C_{\text {salt }}^{-1 / 3}
$$

where $c_{\text {salt }}$ is the external salt concentration and $\sigma_{b r u s h}$ the grafting density. In this regime the brush thickness decreases with an exponent of minus one third of the external salt concentration.

Linse [99] studied the interaction between colloidal particles carrying grafted AB-diblock polyampholytes using a coarse-grained model with MC simulations. Many features of this system were found to be controlled by the charge of the end-grafted blocks B. In the limit of uncharged B blocks the grafted chains were stretched and formed an extended polyelectrolyte brush, and a strong repulsive force operates between two colloids. In the limit where the charges of the two blocks exactly compensate each other, chains collapsed and formed a polyelectrolyte complex surrounding the cores, and an attractive force appeared between two colloids.

Tamariso et al. [100] presented an analytical treatment, at the MF level, of the ionic-strength dependence of the normal forces between two opposing quenched polyelectrolyte (PE) brushes under 
compression. The authors compared the theoretical predictions with experimental measurements carried out on PE brushes formed by the adsorption of diblock copolymers PtBS/ NaPSS onto hydrophobized mica [101]. Despite many simplified theoretical assumptions, when the anisotropic polymer profile was taken into account the comparison displayed a reasonable quantitative agreement at high salt concentration [100]. On the other hand, results obtained for isotropic brushes were found to describe better the high-compression regime, when the opposing PE chains could overlap.

Polydisperse brushes. Milner et al. [102] consider the effects of polydispersity in molar mass on the equilibrium statistics of the grafted polymer brushes. The authors approximated the configurations of strongly stretched polymers in a SCF as their most probable configuration. A general solution for the density and pressure profile and the force required to compress a brush were given in terms of the distribution of molecular masses. The density profile was found to be softened at its outer extremity by the addition of some longer polymer chains and made steeper near the grafting surface by the addition of shorter chains. So, the assumption of a block profile is even less accurate for polydisperse brushes.

\section{Techniques}

\subsection{Surface Force Apparatus (SFA)}

The SFA was developed approximately 40 years ago by Tabor and Winterton [103] to measure forces between two cylinder surfaces in air or vacuum. Later, an adjusted version of the apparatus which enables measurements in liquids was constructed by Israelachvili and Adams [104, 105]. Figure 8 shows a schematic sketch of the apparatus which consists of two mica surfaces in a crossed cylinders configuration, the interactions between which can be measured using highly sensitive force-measuring springs [7]. The separation distance between the cylinder's surfaces can be measured optically, applying a spectrometer, from multiple beam interference fringes. Moreover, the spectrograms give the exact shapes of the two surfaces and the refractive index of the solvent between them. The latter allows to determine the quantity of material deposited or adsorbed on the surfaces [7]. The SFA can be used with turbid materials as well with some extensions: using a capacitance method instead of the 
optical separations measurement [106]. The positioning of the cylinders can be done using a threestage system, consisting of springs and a piezoelectric tube, which allows to adjust the distance with different precision levels (from $1 \mu \mathrm{m}$ to $0.1 \mathrm{~nm}$ ).

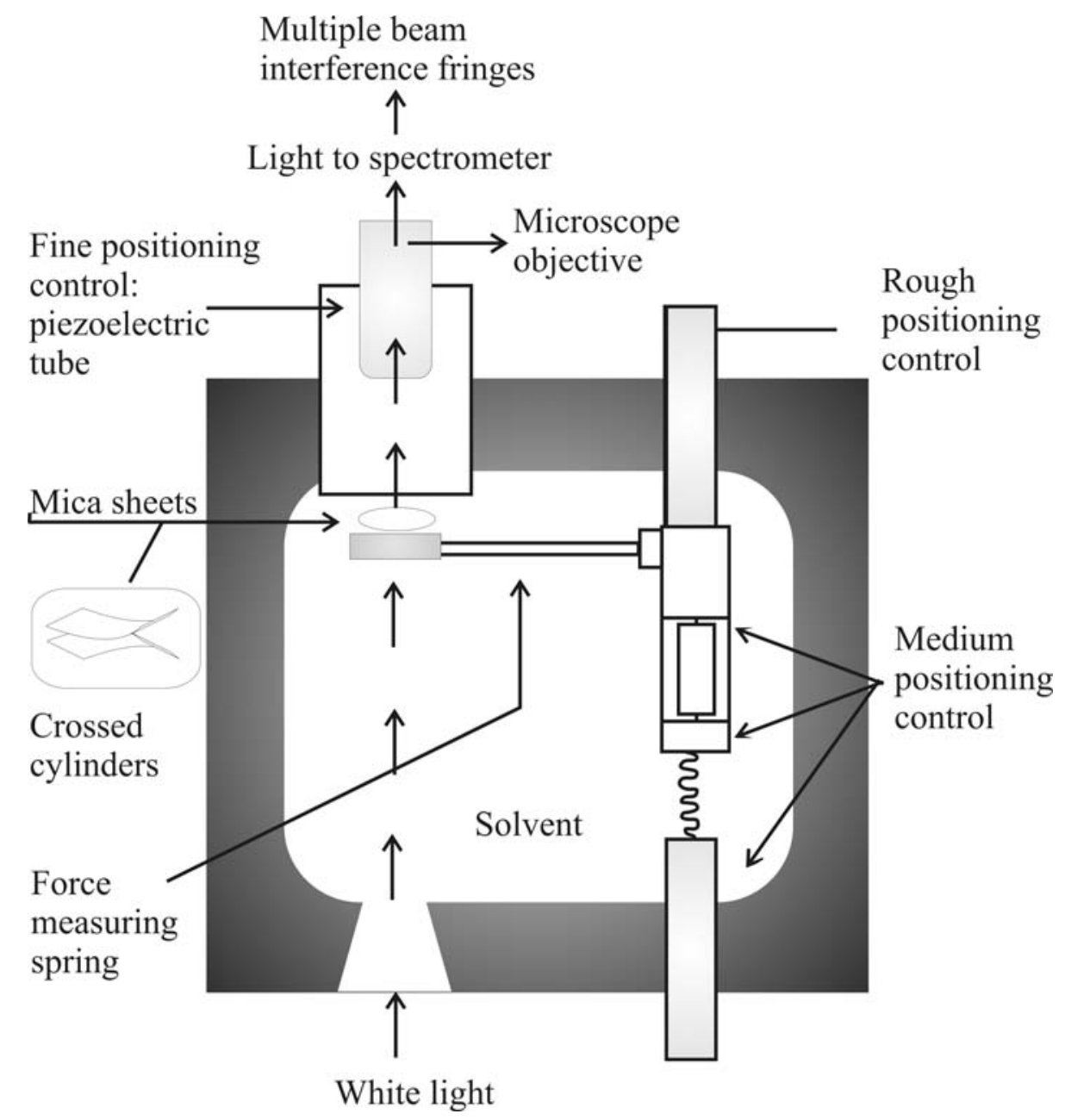

Figure 8. Schematic picture of the SFA. Two mica sheets in crossed cylinder configuration are positioned with the precision of $0.1 \mathrm{~nm}$ using rough, medium and fine positioning control. The light passes through the cylinder surfaces to the spectrometer. Multiple beam interference fringes from the spectrogram provide the separation distance.

An SFA force measurement is performed by moving the top mica surface for a certain known distance and monitoring the actual deflection of the bottom surface, $h^{*}$, as a function of final separation, $h$. The difference $\Delta=h-h^{*}$ multiplied by the stiffness of the force-measuring spring provides the force acting between the surfaces at separation $h$. Thus, the force vs. distance curve can be obtained for attractive as well as for repulsive interactions. The force between two cylinders is scaled with their radius of curvature to enable comparison between different measurements. 
Advantages. As the main advantage SFA offers an extremely high spatial resolution of $0.1 \mathrm{~nm}$. The distance resolution in other techniques (AFM, TIRM, optical tweezers) is limited by the roughness of the probe surface [13]. Characteristically, SFA uses atomically smooth mica sheets.

Disadvantages. High contact area of $1 \mu \mathrm{m}^{2}$ and low force resolution in the range of $10^{-8} \mathrm{~N}$. Further, SFA is limited to the measurement of forces between model surfaces and cannot be directly applied to colloidal particles.

\subsection{Atomic force microscope (AFM)}

The atomic force microscope (AFM) was designed to obtain high-resolution topographical and force analysis, applicable to both conducting and insulator surfaces [107]. The basic measuring principle is very simple (see figure 9): a sample attached to a piezoelectric positioner is scanned by a sharp tip attached to a sensitive cantilever spring. Forces between the tip and the sample lead to deflection of the spring, which is monitored optically [108]. A topographic image of the sample is obtained by plotting the deflection of the cantilever vs. its position on the sample. Alternatively, a feedback loop can be used to hold the spring deflection constant, and the corresponding movement of the piezoelectric positioner thus generates the image [12]. Interactions present in colloidal systems can be studied with a colloidal probe colloidal particle $(2-20 \mu \mathrm{m}$ diameter) which is attached to the end of the cantilever AFM (CP-AFM) [109]. 


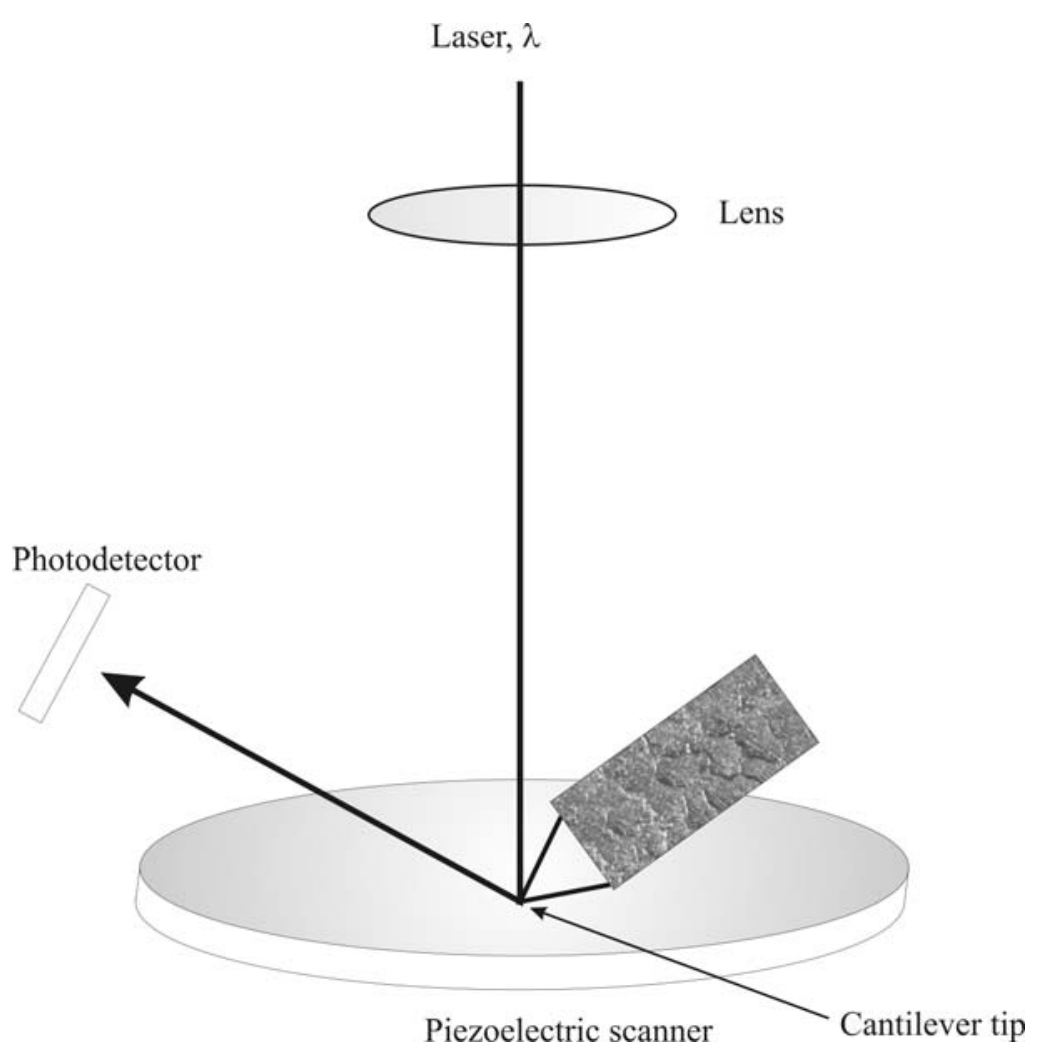

Figure 9. Schematic picture of an AFM. The sample is placed on the piezoelectric scanner. A laser is reflected off the upper side of the cantilever and into a split photodetector. In this way, vertical and horizontal deflection signals can be measured. For CP-AFM, a well-defined colloidal particle is glued to the tip of the cantilever.

An AFM-CP force measurement is performed by brining probe and substrate together and monitoring cantilever deflection as a function of displacement. The photodetector voltage vs. piezo position curve can be converted into a force vs. distance curve. The force acting on the cantilever is obtained by multiplying the known spring constant of the cantilever with its deflection. The zero force line defines zero deflection of the cantilever (when the colloidal probe is far from the surface of the substrate). To obtain the force-distance dependence on an absolute scale the zero distance should be determined. In AFM the zero distance is obtained from the force curve itself and not through an independent method [12]. Practically, a region where the particle and the wall come into physical contact (then the probe movements complies with the movement of the piezo, a region of constant compliance) defines the point of zero distance. In most applications this is correct and causes no significant problem. In some cases, particularly relevant for polymer-induced interactions, this method 
may raise a false interpretation. Especially in the case of highly deformable surfaces, when the probe may contact the sample before constant compliance occurs.

Force-distance curves recorded with the AFM CP are dependent on the specific geometry of the probe and the surface, which were used in the experiment. To enable comparison between different measurements commonly, the measured force is normalized by the known particle radius. Usually, the interaction is displayed as the force divided by the radius of the colloid $R$, in units $\mathrm{N} / \mathrm{m}$. The Derjaguin approximation relates this quantity to the interaction potential per unit area between equivalent flat surfaces at given separation distance (see section 2.1. Non-adsorbing polymers (depletion)).

Advantages. Since the AFM is widely used for imaging it is relatively common and the technology is well-developed. Due to its high lateral resolution of $1 \mathrm{~nm}$ small samples can be used and material inhomogeneities can be mapped. Having small contact areas $\left(10 \mathrm{~nm}^{2}\right)$ also reduces the probability of measurement artefacts due to surface contamination and roughness [12]. The high spatial resolution capability makes AFM a complementary approach to the SFA which has been used to measure interfacial forces between proximal surfaces over areas on the order of $1 \mu \mathrm{m}^{2}$. Moreover, the force resolution of AFM is higher than that of the SFA.

Disadvantages. The determination of the zero separation distance remains difficult in some cases, particularly relevant for polymer-induced interactions. Low force sensitivity.

\subsection{Total Internal Reflection Microscopy (TIRM)}

The interaction potentials between a single particle and a wall can be obtained using evanescent field scattering in total internal reflection microscopy (TIRM) [13, 14]. This technique makes use of Boltzmann's law which connects the probability density to find the particle at a certain separation distance, $h$, with its potential energy at this distance, $\phi_{\text {tot }}(h)$ :

$$
p(h)=C \exp \left(-\frac{\phi_{t o t}(h)}{k_{B} T}\right)
$$

where $C$ is a normalization constant. 
The fluctuations of the separation distance resulting from thermal motion can be directly observed by TIRM. For this purpose a laser beam is directed via a prism to the glass/solution interface as sketched in figure 10, with an incident angle, $\alpha_{i}$, such that it is totally reflected. The electric field of the laser beam penetrates the interface causing an evanescent wave, the amplitude of which decays exponentially along the normal of the interface. A single colloidal sphere, interacting with this evanescent wave, will scatter light depending on its position, $h$, as [110]

$$
I_{s}(h)=I(h=0) \exp \{-\xi h\}
$$

where $\xi$ is the inverse penetration depth of the evanescent wave. A photomultiplier is used to monitor the scattered intensity as a function of time, with a resolution in the ms-range. For a sufficiently high number of data points (typically more than $5 \cdot 10^{4}$ ) the histogram of intensities converges to the probability density distribution of the intensity. By virtue of equation (19) the latter is directly related to the probability density of separation distances which can be converted into a potential energy profile using Boltzmann's law (equation (18)). Additionally, an optical trap can be built in to prevent the colloidal particle from moving out of the microscope's observation area. For this purpose a second laser beam has to be focused directly at the particle.

As was shown recently [111], equation (19) may be violated if inappropriate penetration depths and/or polarization of the incident beam are applied. It is recommended to use p-polarized light and a penetration depth below $\xi^{-1}<150 \mathrm{~nm}$. 


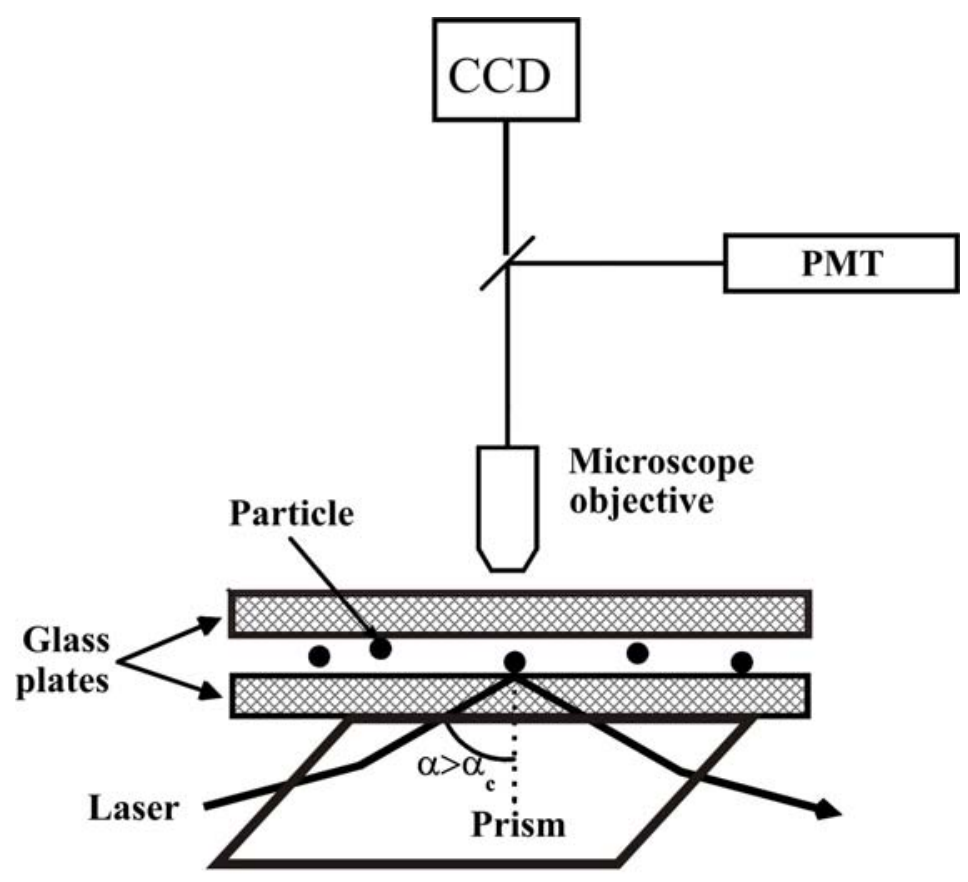

Figure 10. Sketch of a TIRM. If the incident angle, $\alpha_{i}$, is larger than the critical angle the incident beam is totally reflected at the glass-fluid interface and the evanescent wave penetrates into the fluid. A colloidal particle located close to the surface will scatter light from the evanescent wave, which is collected by a photomultiplier and provides the probability density of separation distances between the particle and the wall. A CCD camera is used to image the field of view.

Advantages. Major advantages of this technique relative to AFM and SFA for studying interaction potentials are its extreme force sensitivity and its non-invasive nature. With TIRM it is possible to investigate the interactions of a single, freely moving, Brownian particle. This method enables measurements of forces as small as $10^{-14} \mathrm{~N}$, the reason for this extreme sensitivity is the use of a molecular gauge for energy $\left(k_{B} T\right)$ instead of a mechanical gauge for the force determined by a spring constant, as it is used in AFM and SFA [13]. TIRM is the technique which enables to obtain the interaction potential between the colloidal particle and a wall.

Disadvantages. It is impossible to measure large interaction potentials with TIRM. If the repulsion between the particle and the wall does not fall below $\sim 5 k_{B} T$ within the penetration depth of the evanescent wave, the probability density to find the particle in this range becomes virtually zero. Therefore, the error in determination $p(h)$ becomes very large. Further, if the attraction between the 
sphere and the wall becomes too strong, the intensity histogram becomes narrower than the range set by the electronic noise of the photomultiplier [13]. In such cases it is not possible to determine interaction potentials with reasonable accuracy using TIRM.

\subsection{Optical Tweezers}

Almost 40 years ago Ashkin [112] found that laser radiation forces can be used to trap and manipulate small dielectric particles. A weakly focused laser beam will push a particle towards the centre of the beam, if the particle has a higher refractive index than the surrounding medium. Thus, optical tweezers allow to pick up and manipulate colloidal particles in 3D-space. This technique found a broad application in biology as well as in colloid science $[113,114]$.

Figure 11 shows a simple optical tweezers arrangement. The laser beam is tightly focused using the microscope objective lens, which also gives the possibility to image trapped particles with a camera. Optical tweezers can be configured using multiple beams to trap many particles simultaneously. This has been implemented by [10]: i) the rapid scanning of a single beam between two or more trap positions, ii) splitting the beam at an early stage in the optical circuit to produce two separate light paths which are then recombined before entering the microscope and iii) using computer-generated holograms to give multiple beams simultaneously. 


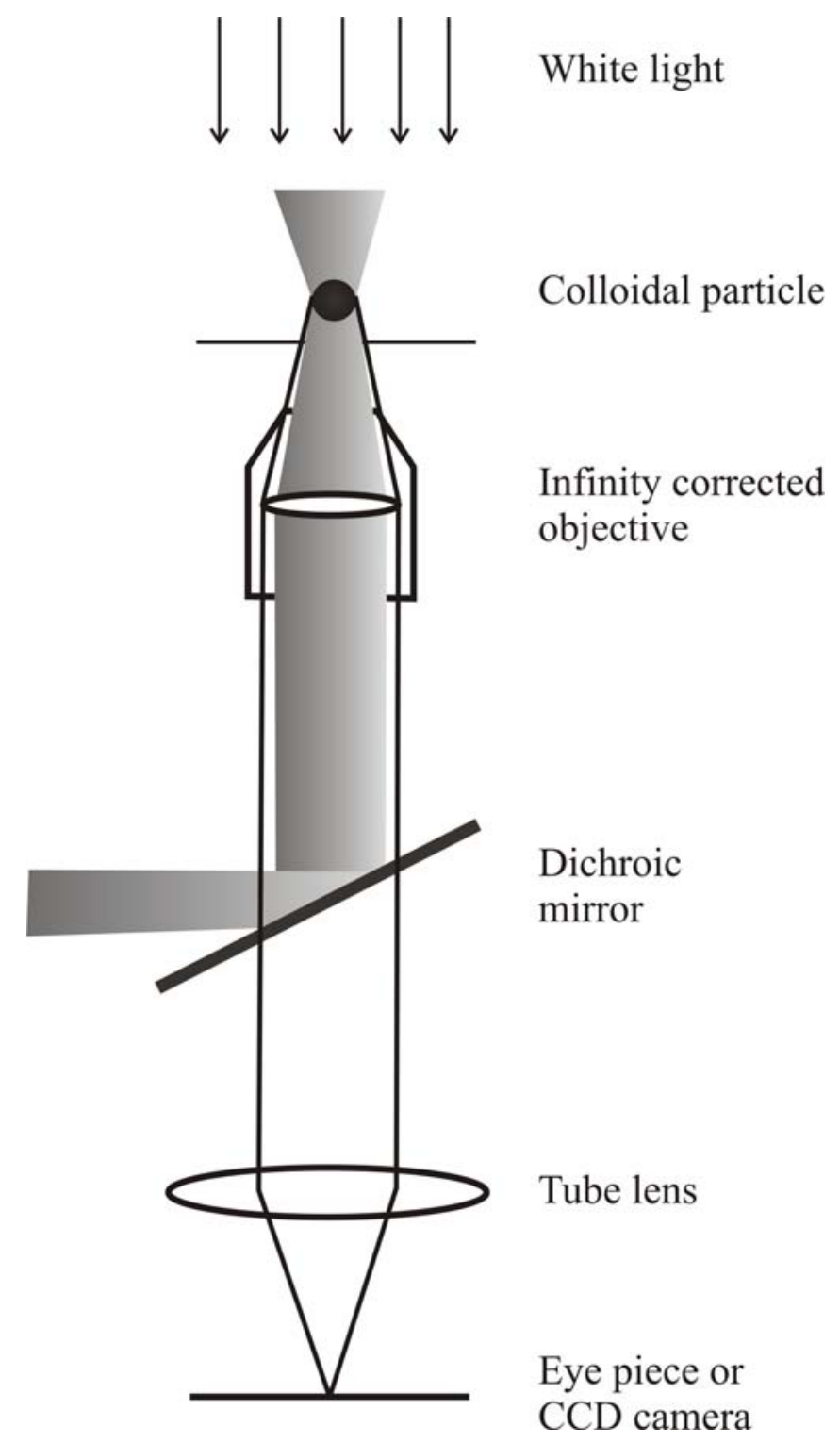

Figure 11. A sketch of a simple optical tweezers arrangement. The microscope objective lens enables the tight focusing of the laser beam and imaging of trapped particles.

Boltzmann's law (equation (18)) is used to find the interaction potential between the trapped particles using the measured probability density as a function of separation distance. Two methods can be used for the position detection: i) particle tracking from video microscopy and ii) back focal plane interferometry [8]. The first method requires the acquisition of bright field or fluorescent images from the microscope [115]. Particle centre separations can be determined then with a sub-pixel resolution through image-processing operations $[115,116]$. A spatial resolution of $\sim 10 \mathrm{~nm}$ can be achieved [116]. To improve this, back focal plane interferometry is an alternative [117]. In that case, the laser beam from the trap is imaged onto a quadrant photodiode, resulting in an interference pattern that is 
used to determine the particle position relative to the trap. The spatial resolution is significantly improved then to $\sim 1 \mathrm{~nm}$.

Advantages. A major advantage of optical tweezers is, as in the case for TIRM, the force sensitivity (down to $10^{-13} \mathrm{~N}$ level with the resolution of $5 \cdot 10^{-14} \mathrm{~N}$ ). Additionally, it is possible to measure large forces up to $2 \cdot 10^{-10} \mathrm{~N}$. Optical tweezers allow as well the manipulation of particles by exerting forces on the $10^{-12} \mathrm{~N}$ level with a high precision in force $\left(10^{-12} \mathrm{~N}\right)$ and space. Moreover, optical tweezers enable studying colloidal interactions in a non-invasive manner. Complementary to TIRM it enables to measure the interaction potentials between two colloidal particles.

Disadvantages. The measurements with optical tweezers can be susceptible to misinterpretations due to image processing problems $([118,119])$.

\section{Analysis of measured forces}

\subsection{Depletion}

The magnitude of the depletion interaction at contact between colloidal spheres in a solution of ideal polymer chains is $\phi_{\text {depl,sphere-sphere }}(h=0) / k_{B} T=-3 \ln 2 \cdot\left(c / c^{*}\right) \cdot\left(a / R_{g}\right)$ [62]. Here $c_{p}$ is the polymer mass concentration in $\mathrm{g} / \mathrm{L}$, the polymer overlap concentration $c^{*}$, related to the molar mass $M$ and the radius of gyration $R_{g}$ of the polymer as $c^{*}=3 M / 4 \pi R_{g}^{3} N_{A}$. In a realistic situation for the direct interaction potential measurements the polymer concentration $c=0.1 \cdot c^{*}$ and the ratio $R_{g} / a$ of 0.03 , corresponding to the polymer radius of gyration $\sim 30 \mathrm{~nm}$ and a sphere radius of $\sim 1000 \mathrm{~nm}$, the resulting small value of $\Delta \phi_{\text {depl }}(h=0) \sim 7 k_{B} T$ illustrates why direct measurements of depletion interaction in polymer solution are experimentally challenging. Therefore, it is not surprising that depletion was first measured directly only fifteen years ago with SFA [120] and AFM [121]. However, these first measurements were performed either with charged micelles as depletants [120] or in concentrated polymer solutions [121] in order to increase the magnitude of the depletion interaction. 
Luckham and Klein were one of the first who tried to measure the depletion interaction directly [122]. They applied SFA to study depletion forces between two mica cylinders due to non-ionic polystyrene (PS) chains in toluene at good solvent conditions, when adsorption of PS on mica was not favourable [123]. However, the depletion forces were too weak to be detected by SFA. As the authors conjectured, the calculated contact value $\left(\sim 4 \mathrm{nJm}^{-2}\right)$ was at least 2-3 orders of magnitude smaller than the inherent detection limit of the apparatus. Further, the same authors studied depletion interaction in an aqueous solution of polyethylene glycol (PEO), a neutral polymer for which water is a good solvent [124]. Their mica surfaces were covered with adsorbed Triton X-100 chains so as to prevent adsorption of PEO. Again, no attractive force was detected. The authors explained this finding by suggesting, that PEO chains from the solution replace the surfactants molecules from the mica surfaces causing steric repulsion.

A crossover from an attractive depletion interaction to repulsion due to adsorbed polymers was shown by Kuhl et al. [125-127] using SFA force measurements between lipid bilayers (consisting of dipalmitoyl phosphatidylethanolamine (DPPE) and dimyristoyl phosphoatidylcholine (DMPC)) adsorbed onto the mica cylinders in aqueous solutions of polyethylene oxide (PEO) $\left(M_{w}=1000-\right.$ $20000 \mathrm{~g} / \mathrm{mol}$ ). It was found that PEO with a molar mass lower than $6 \cdot 10^{3} \mathrm{~g} / \mathrm{mol}$ does not have a large enough size to generate a significant depletion force, while high molecular mass PEO $\left(M_{w}>18000\right.$ $\mathrm{g} / \mathrm{mol}$ ) adsorbs sufficiently onto the bilayer surfaces to suppress depletion attraction quantitatively and to cause a repulsive steric barrier as shown in figure 12. Only in PEO solutions with $M_{w}=8000 \mathrm{~g} / \mathrm{mol}$ an attractive depletion force was observed. Using scaling arguments [51] the authors estimated the depletion layer thickness to be $\delta=14 \AA$, which was in good agreement with an experimental value of $2 \delta=25 \pm 5 \AA$. Using this length scale and equation (1) the experimental results for depletion interaction in figure 12 were converted to a bulk osmotic pressure and compared to a value from the literature. The experimental value was found to match very well with the literature data for the bulk osmotic pressure. As one can see from figure 12, a very weak repulsion was measured with PEO 8000 at separations larger than those where depletion attraction occurs. The authors attributed the origin of this repulsion to the presence of high molecular mass PEO chains in a polydisperse, commercial grade PEO 8000 sample. 


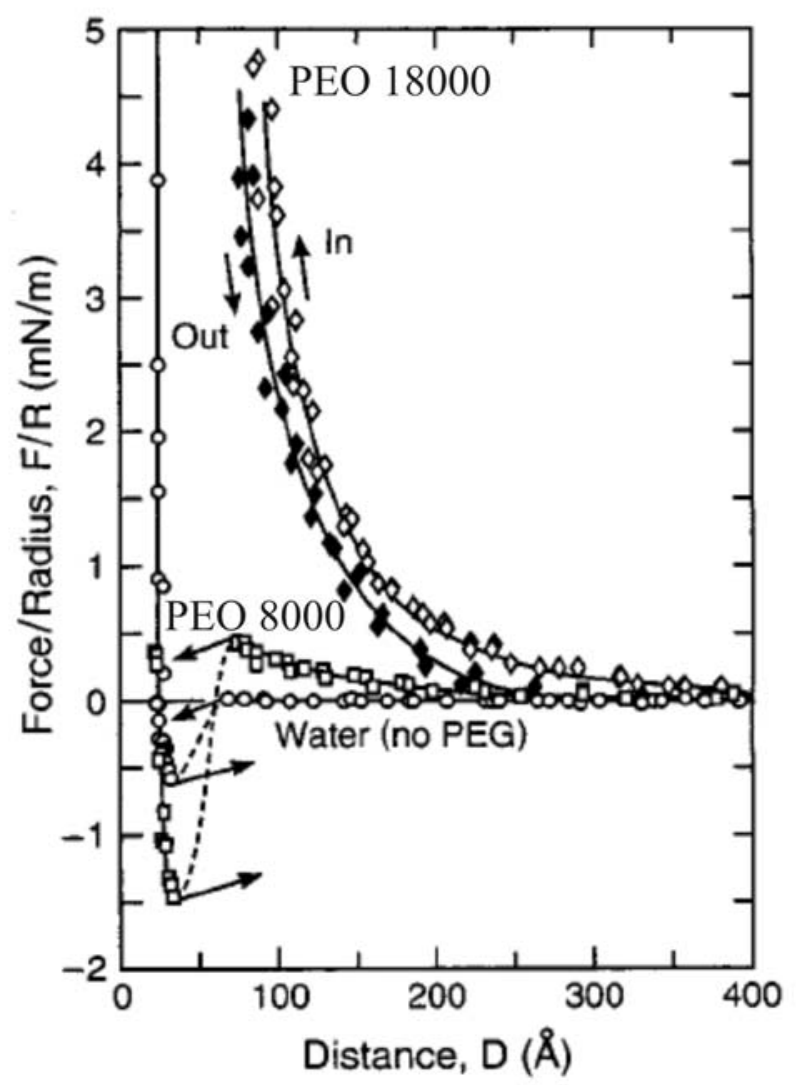

Figure 12. Force profiles of DPPE/DMPC bilayers in water and aqueous PEO solutions obtained by Kuhl et al. [127]. The circles and dashed curves are the force profile in pure water, where the bilayers attract due to van der Waals forces. The arrows indicate when the spring constant is exceeded by the gradient of the attractive force. The resulting mechanical instability causes the surfaces to jump together or apart. Thus, these parts of the force profile are inaccessible. Squares are the force profile in $10 \%$ solution of PEO 8000 . In this case the attraction between the surfaces is significantly larger due to depletion attraction. Open diamonds are the force profile taken upon the approach in PEO 18000, while the filled diamonds were taken during separation. Due to the adsorbed PEO 18000 on the bilayer surface a strong steric repulsion was found. The hysteresis upon approach and separation of the surfaces was characteristic of adsorbed PEO layers in water $[122,128]$. The lines are guides to the eye. Reprinted figure with permission from: Kuhl T. L., Berman A. D., Hiu S. W., Israelachvili J. N. 1998 Macromolecules. Copyright 1998 by the American Chemical Society.

Rudhardt et al. $[129,130]$ performed TIRM measurements on the interaction between a charged glass plate and a charged polystyrene (PS) sphere with radii 1.5 and $5 \mu \mathrm{m}$ in the solutions of PEO with 
$M_{w}=1 \cdot 10^{6}$ and $2 \cdot 10^{6} \mathrm{~g} / \mathrm{mol}$, measurements under similar conditions were performed by Ohshima et al. [131] using laser radiation pressure. A strong attractive contribution to the interaction potential was found. The experimental potential profiles were analyzed using the Askura - Osawa model (equation (4)), in which the polymers are approximated as phantom spheres. Non-linear least squares fitting yielded $R=107 \mathrm{~nm}$ and $150 \mathrm{~nm}$ for the phantom sphere radii, the latter of which is in excellent agreement with the value obtained by Ohshima et al. with a different experimental approach.. However, these values were clearly larger than the radii of gyration of PEO, $R_{g}=67.7 \mathrm{~nm}$ and $101 \mathrm{~nm}$, the authors reported. Different from the work by Rudhardt et al. and Ohshima et al., no depletion interaction was observed in the same system by Kleshchanok and Lang using TIRM [132]. This shows that the question whether PEO absorbs on surfaces (thereby causing steric repulsion) or whether it is depleted from interfaces (thereby causing attraction), is a delicate issue, depending on very subtle details of sample history and preparation (see also section 4.2. Forces induced by attached polymers).

Non-ideal depletants (polymers, micelles, spheres, rods). As it was already predicted by Asakura and Oosawa in 1958 [24] charges on polymers increase the range and the absolute value of the depletion interaction. This is the reason why first successful direct measurements on depletion interaction were performed with charged depletants. SFA measurements by Richetti and Kekicheff $[120,133]$ of depletion attraction due to cetyltrimethylammonium bromide (CTAB) micelles at high volume fractions showed oscillatory force profiles, with the number of oscillations per separation distance and their magnitude increasing with the CTAB concentration. Similar measurements by Sober and Walz [134] using TIRM also demonstrated depletion attraction in the presence of CTAB micelles. However, their micelle concentration was much lower than in the experiments conducted by Richetti and Kekicheff and no oscillations in the force were detected. The reason for the oscillations in the interaction potential might be so-called structural forces, which may occur due to free energy changes upon packing of charged micelles in the confined space between approaching surfaces. Biggs et al. [135] used the oscillations in the depletion potential caused by the presence of polyelectrolyte sodium polystyrene sulfate (NaPSS) measured both by TIRM and AFM to calibrate the AFM data a posteriori. Later, Jönsson et al. [136] performed MC simulations and density functional calculations for charged 
macromolecules (polyelectrolytes, micelles, spheres) confined in planar slits. The force between the walls had been evaluated as a function of separation, while keeping the chemical potential of the charged depletant constant. The authors found, in agreement with experiments [137], that highly charged spheres and flexible polyelectrolyte chains in confinement give rise to depletion and structural oscillatory forces as a function of surface separation. The net charge, the range of interaction, and the particle density affected the details of the force curve. For spherical depletants, the period of the oscillations was detected to scale approximately with their bulk concentration as $c_{b u l k}{ }^{-1 / 3}$. It was found that polyelectrolyte chains pack as cylindrical objects and not as spheres; therefore, the effective repulsive interaction between polyelectrolyte chains can be more long-ranged and oscillatory forces can appear more readily than for a corresponding solution of equally charged spherical macroions.

Most synthetic and natural polymers do not consist of monodisperse chains but have a finite molar mass distribution. The effect of polymer polydispersity on depletion interaction between a charged PS sphere and a charged glass wall induced by dextran, a non-adsorbing polydisperse polysaccharide was studied by Kleshchanok et al. [62] using TIRM. The polymer size polydispersity was shown to greatly influence the depletion potential. No quantitative agreement between the experimental profiles and the simple PHS approximation for monodisperse polymers was found (equation (5)). The dashed lines in figure $\mathrm{Da}$ ) were calculated (applying the Derjaguin approximation to obtain $\phi_{\text {depl, sphere-plate }}$ ) using the zaveraged radius of gyration of dextran, $\left\langle R_{g}\right\rangle_{z}$ and its weight-averaged molar mass, $M_{w}$, both determined by static light scattering. Clearly, the PHS model significantly overestimates the range and depth of the depletion potential. This is due to the fact that the polydisperse dextrans used in the experiment could not be described as monodisperse chains with a single characteristic polymer size. Substitution of $R_{g}$ into equation (5) with the weight-average radius of gyration $\left\langle R_{g}>_{w}\right.$ provided a better match. Nevertheless, the depletion potential range and depth were still predicted too large. Using theory for the depletion interaction due to ideal polydisperse polymer chains [61], with known molar mass distribution (equation (9)) allowed an accurate description of experimental data with only a single adjustable parameter, which depends on the segment length and the polymer chain architecture. The results are presented as full curves in figure 13c). To conclude, a satisfactory description of the depletion potential is not always possible using averaged values of the radius of gyration. For 
polymers with a broad molar mass distribution the full distribution has to be incorporated into the theoretical expressions for $\phi_{\text {depl }}(h)[62]$.

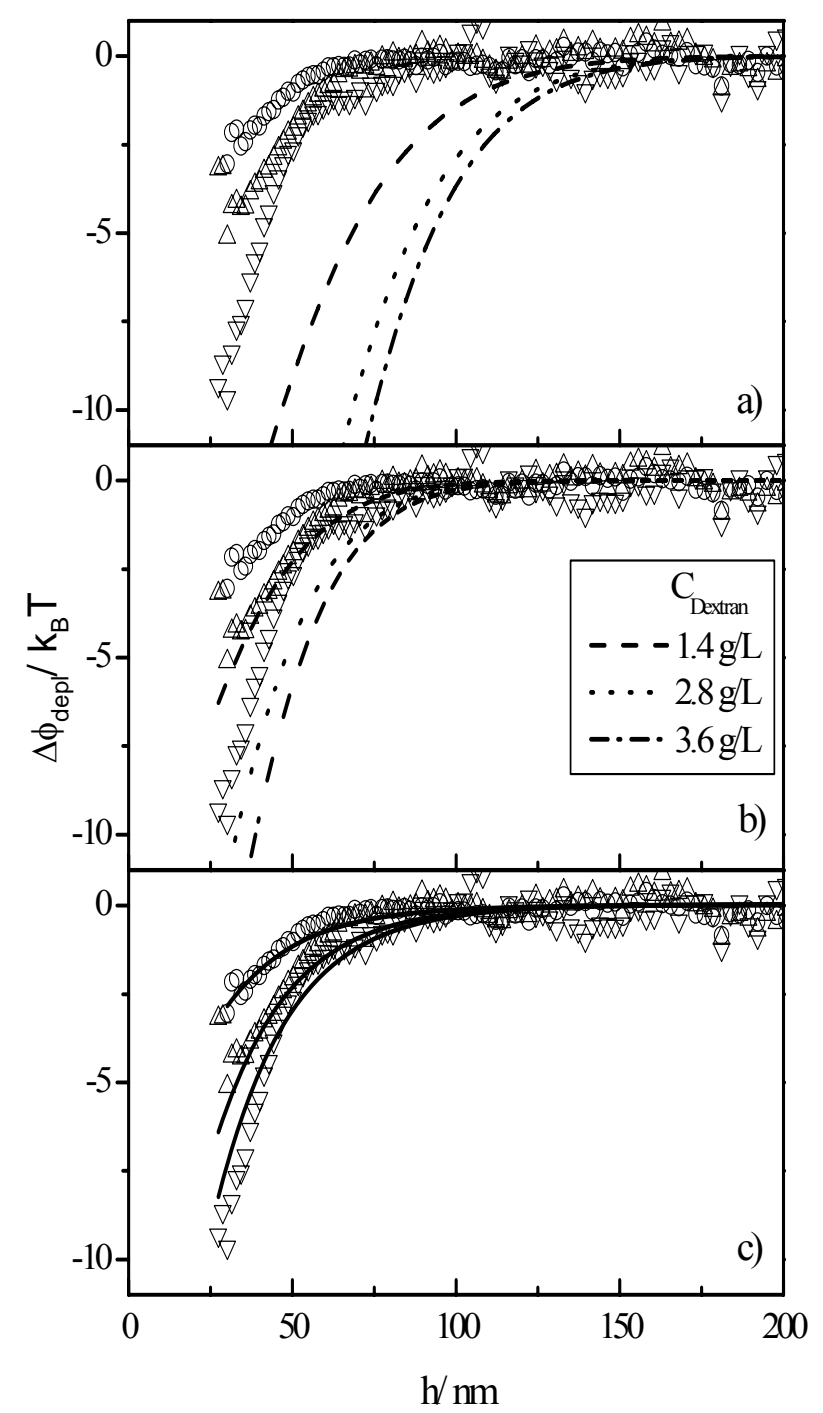

Figure 13. Depletion potential between a $5.7 \mu \mathrm{m}$ diameter polystyrene sphere and a glass wall in aqueous solutions of dextran, with: $\bigcirc c=1.4 \mathrm{~g} / \mathrm{L} ; \Delta c=2.8 \mathrm{~g} / \mathrm{L} ; \nabla c=3.6 \mathrm{~g} / \mathrm{L}$. The curves are: a) model calculations using the PHS model for a monodisperse polymer with known $\langle R g\rangle_{z}$ and $M_{w}$; b) model calculations using the PHS model for a polydisperse polymer with known $\left\langle R g>_{w}\right.$ and $M_{w}$; c) non-linear least squares fits with equation (9) using the full molar mass distribution of the polymer [62]. Reprinted figure with permission from: Kleshchanok D., Tuinier R., Lang P. R. 2006 Langmuir. 22 9121. Copyright 2006 by the American Chemical Society. 
Experiments on depletion interactions in solution with polymer concentrations near and above the overlap concentration, where interactions between polymer segments become important, were performed by Verma et al. $[116,138]$. Scanning optical tweezers were used to study the depletion potential between two silica spheres with a diameter of $1.25 \mu \mathrm{m}$ in a solution of rather monodisperse DNA with an averaged radius of gyration of $500 \mathrm{~nm}\left(\mathrm{c}^{*}=96 \mu \mathrm{g} / \mathrm{mL}\right)$ and screened Debye length of 3 nm. In figure 14 their results are reproduced for concentrations of $140 \mu \mathrm{g} / \mathrm{mL}$ (solid squares), $190 \mu \mathrm{g} / \mathrm{mL}$ (open circles) and $280 \mu \mathrm{g} / \mathrm{mL}$ (solid circles). Thus, the authors found that the order of magnitude of measured attraction can be compared reasonably well to the results from the PHS theory but the range of attraction was overestimated by this theory. For polymer concentrations above the overlap concentration one should take into account the non-ideality of the polymer solution. Thus, taking into account the excluded volume interactions between polymer segments [42] gives the full curves presented in figure 14 (also see section 2.1.2 Non-ideal depletion cases). The agreement with the experiment is now much better.

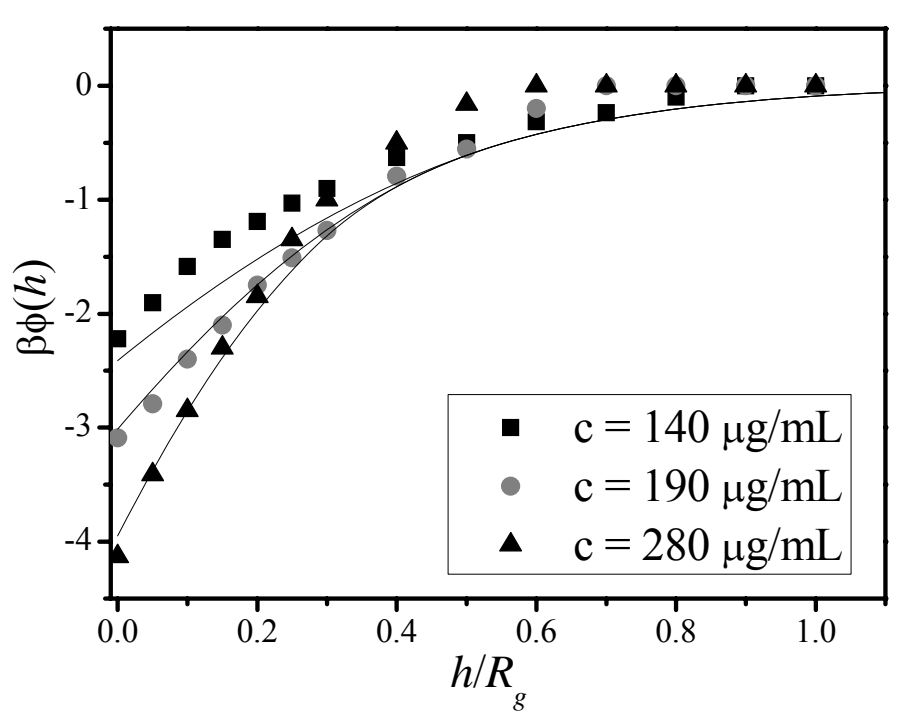

Figure 14. Interaction potentials between $\mathrm{SiO}_{2}$ spheres mediated by DNA segments, data by Verma et al. $[116,138]$. Measured interaction potentials are represented by the symbols. The results of the theory, which takes into account the excluded volume interactions between 
polymer segments [42], are given by the full curves. Polymer concentrations are indicated in the plot.

Lin et al. [139] studied the depletion interactions of colloidal spheres in suspensions of rod-like fdviruses using line-scanned optical tweezers. The influence of sphere size, rod concentration, and ionic strength on these interactions was investigated. The results were compared with different models: i) the model of Yaman, Jeppesen and Marques (the YJM model), which applies to any size ratios $a / L$ [70] (also see section 2.1.2 Non-ideal depletion cases); ii) the model derived by Mao et al. [66, 67] valid in the Derjaguin approximation (equation (14)), the authors refer to as the Derjaguin model; iii) the Asakura-Oosawa (AO) model for the depletion due to rigid spherical macromolecules (equation (4)). The results are reproduced in figure 15 for a rod concentration of $0.7 \mathrm{mg} / \mathrm{mL}$ (symbols). It is clear that the Derjaguin model (dashed curve) overestimates the experimental interaction potential. The AO sphere model (ditched curve) was rescaled by the authors to match with the potential at contact with $\mathrm{L}$ $=0.5 \cdot a$. One can see from figure 15 that the rods produce a depletion interaction more than 1000 times stronger than the same volume fraction of spherical depletants. Thus, they are very efficient depletants. The YJM model was found to predict approximately the correct magnitude and shape of the depletion potential. The experimental deviations from the YJM model were attributed by the authors to the entropy associated with rod flexibility [140]. At moderate electrolyte concentrations the solution Debye length was found not to influence the depletion potential, which supports the validity of the superposition approximation (see section 2.1.2. Non-ideal depletion cases). Only at very high salt concentration the interaction turned to be repulsive due to bridging of the spheres by fd-rods. 


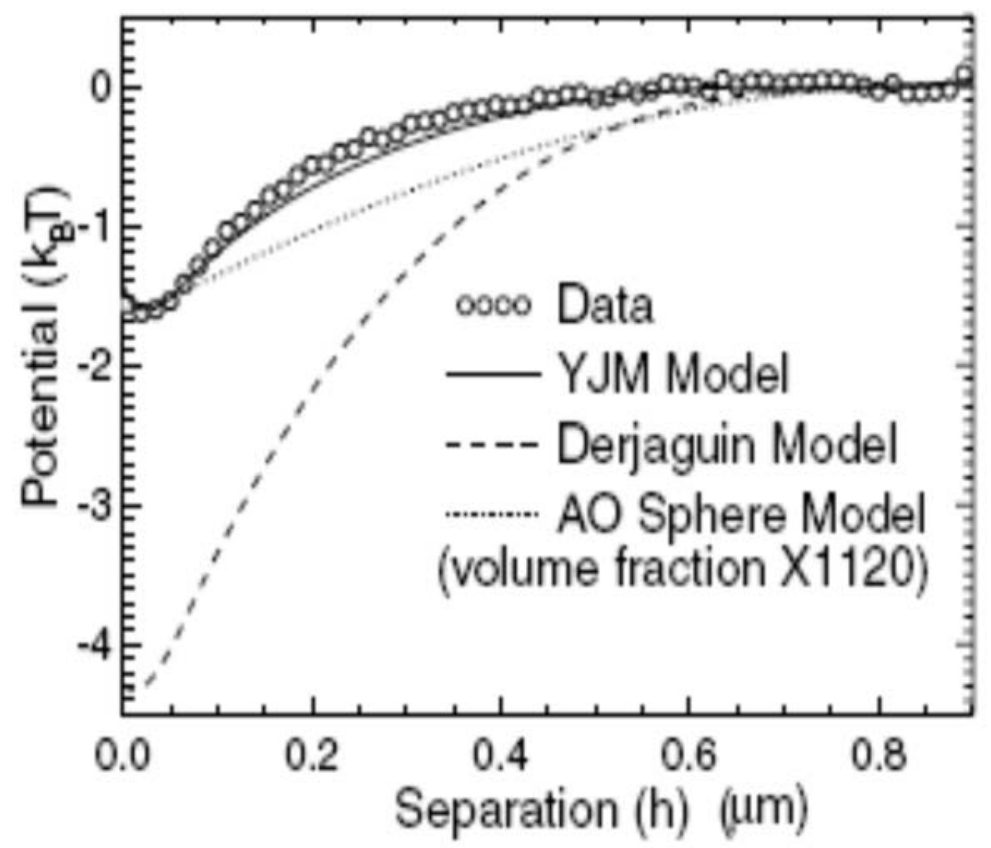

Figure 15. Interaction potential between two spheres $(a=0.5 \mu \mathrm{m})$ in fd-virus suspension at concentration $0.7 \mathrm{mg} / \mathrm{mL}$ [139]. Measured interaction potentials are represented by the symbols. The lines are three different theoretical models indicated in the plot. Reprinted figure with permission from: Lin K, Crocker J C, Zeri a C and Yodh a G 2001 Phys. Rev. Lett. 87 088301. Copyright 2001 by the American Physical Society. http://prola.aps.org/abstract/PRL/v87/i8/e088301

\subsection{Forces induced by attached polymers}

\subsubsection{Physically adsorbed polymers}

Steric repulsion due to adsorbed polymer layers in good solvent conditions was studied by Owen et al. [141] using line-scanned optical tweezers. The pair interaction potential between two silica $\left(\mathrm{SiO}_{2}\right)$ spheres $(a=0.6 \mu \mathrm{m})$ induced by adsorbed PEO chains had been measured. A long-range steric repulsion (range: $\sim 4 R_{g}$ ) was found for the range of potentials $\left(0.1 k_{B} T-5 k_{B} T\right)$ and polymer molar masses $\left(4.52 \cdot 10^{5}-1.58 \cdot 10^{6} \mathrm{~g} / \mathrm{mol}\right)$ to be exponential. The authors modeled the interaction potential 
with an exponential function with a characteristic decay length close to $0.6 R_{g}$. Further, Braithwaite $e t$ al. [142] investigated the adsorption of $5 \cdot 6 \cdot 10^{4} M_{w}$ PEO onto glass in aqueous system using AFM. The authors described the evolution of the structure of the adsorbed polymer layer with time and the resulting variations if only a single surface was allowed to adsorb polymer. The development of the layer was found to change with time from an initially thin layer coverage up to a stable equilibrium layer of approximately $90 \mathrm{~nm}$ thickness. At partial polymer coverage a weak attraction was occasionally observed on approach of the surfaces, which the authors attributed to bridging of the polymer between the two surfaces. At full polymer coverage, repulsive interactions at all surface separations were observed.

Kleshchanok and Lang used TIRM [132] to measure steric repulsion between PEO layers adsorbed on a PS particle and a glass wall. Figure 16 demonstrates the experimental interaction potentials between a $5.7 \mu \mathrm{m}$ sphere and a wall in solution of PEO. The potentials were fitted with a summation of electrostatic repulsion, gravity and brush repulsion (Alexander-de Gennes expression, equation (15)). The authors found that the brush density, $\sigma_{b r u s h}$, and the brush height, $H_{\text {brush }}$ increase monotonically with the polymer concentration in solution. These findings indicated that both the strength of the brush repulsion, which is determined by $\sigma_{b r u s h}$, and the range, which depends on $H_{\text {brush }}$, increase with the polymer concentration. 


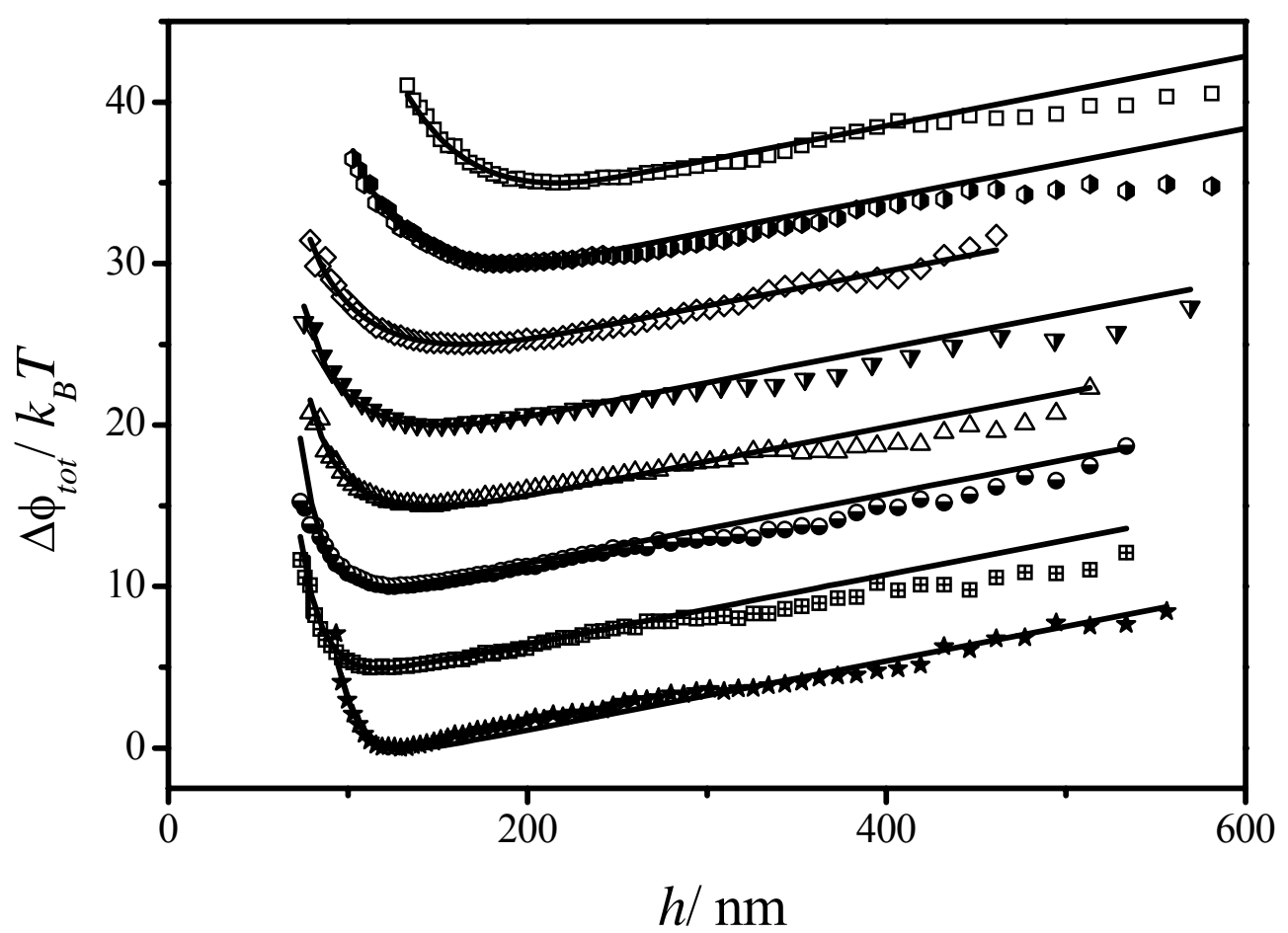

Figure 16. Interaction potentials, $\Delta \phi_{\text {tot }}(h)$, between a $5.7 \mu \mathrm{m}$ diameter PS sphere and a glass wall. Symbols are experimental data obtained at different PEO concentrations: $\star 0 \mathrm{~g} / \mathrm{L}, \boxplus$ $1.5 \cdot 10^{-2} \mathrm{~g} / \mathrm{L}, \bigcirc 2.7 \cdot 10^{-2} \mathrm{~g} / \mathrm{L}, \triangle 4.1 \cdot 10^{-2} \mathrm{~g} / \mathrm{L}, \nabla 8.2 \cdot 10^{-2} \mathrm{~g} / \mathrm{L}, \diamond 1.7 \cdot 10^{-1} \mathrm{~g} / \mathrm{L} ; 3_{3.1} \cdot 10^{-1} \mathrm{~g} / \mathrm{L} ;$

$1.0 \mathrm{~g} / \mathrm{L}$. The solid curves are the best non-linear least squares fits according to a summation of electrostatic repulsion, gravity and brush repulsion [132]. For clarity the individual curves have been shifted vertically by $2 k_{\mathrm{B}} T$ with respect to the curve with the next lower polymer concentration. Reprinted figure with permission from: Kleshchanok D., Lang P. R. 2007 Langmuir. 23 4332. Copyright 2007 by the American Chemical Society.

Pericet-Camara et al. [143] studied interaction forces between pre-adsorbed layers of branched polyelectrolyte poly(ethylene imine) (PEI) of different molecular mass with the AFM-CP. During approach, the long-ranged forces between the surfaces were found to be repulsive due to overlap of diffuse layers down to distances of a few $\mathrm{nm}$. The forces remained repulsive down to contact, likely due to electro-steric interactions between the PEI layers [143]. During retraction of the surfaces, erratic attractive forces were observed which was attributed by the authors to bridging. 
At bad solvent conditions the forces acting between two curved mica surfaces, each bearing a layer of adsorbed PS, immersed in cyclohexane at $24{ }^{\circ} \mathrm{C}$ were studied by Klein [106] using SFA. A zero force was observed at surface separations larger than about $3 \cdot R_{g}$ of the polymer; on closer approach a strong attraction was found to develop between the surfaces, which changed to a repulsion as the surfaces approached closer than about one $R_{g}$.

\subsubsection{Bridging}

Bridging forces between two mica sheets in a cyclohexane solution of poly( $\alpha$-methylstyrene) (P $\alpha \mathrm{MS}$ ) and the kinetics of there evolution were measured by Granick et al. [144] using the SFA. A strong, dominant attraction due to bridging forces was found. The segmental sticking energy of the polymer to mica was estimated by the authors as $k_{B} T / 3$ [144].

Klein and Luckham [145] used SFA to measure the interactions between two smooth mica surfaces immersed in an aqueous solution of PEO (a good solvent system) in the range 0-300 nm apart, and found that at low adsorbance of the polymer on mica there is a reversible, time-independent, longrange $\left(\sim 2.5 R_{\mathrm{g}}\right)$ attraction as the surfaces approach. On permitting equilibrium adsorption of the polymer to take place, the attraction disappeared, to be replaced by monotonically increasing, longrange repulsion [145].

More recently, Goodman et al. [146], used AFM to investigate the influence of grafting density, $\sigma_{\text {brush }}$, and the nature of the monomer on bridging forces. The authors studied the interaction forces acting on latex particles bearing densely grafted polymer brushes which consist of poly(N,Ndimethylacrylamide) (PDMA), poly(methoxyethylacrylamide) (PMEA), poly(N-isopropylacrylamide) (PNIPAM), and PMEA-b-PNIPAM in aqueous media (good solvent). Force profiles of PDMA (0.017 $\left.\mathrm{nm}^{-2} \leq \sigma_{\text {brush }} \leq 0.17 \mathrm{~nm}^{-2}\right)$ and PMEA $\left(\sigma_{\text {brush }}=0.054 \mathrm{~nm}^{-2}\right)$ brushes were found to be purely repulsive upon compression, with forces increasing with $M$ and $\sigma_{b r u s h}$, as expected, due to excluded volume interactions. At a sufficiently low grafting density $\left(\sigma_{\text {brush }}=0.012 \mathrm{~nm}^{-2}\right)$, PDMA exhibited a long-range exponentially increasing attractive force followed by repulsion upon further compression. The longrange attractive force was believed to be due to bridging between the free chain ends and the AFM tip. 
The PNIPAM brush exhibited a bridging force at $\sigma_{b r u s h}=0.037 \mathrm{~nm}^{-2}$, a value larger than the grafting density needed to induce bridging in the PDMA brush. Bridging was therefore found to depend on grafting density as well as on the nature of the monomer. The grafting densities of these polymers were larger than those typically associated with bridging. The occurrence of bridging interactions was interpreted by the authors as strong evidence for the presence of PNIPAM in a block copolymer PMEA-b-PNIPAMA brush given that the original PMEA homopolymer brush produced a purely repulsive force.

\subsubsection{Grafted polymers}

The influence of the layer structure of amphiphilic diblock copolymers of poly(t-butyl styrene) poly(styrene sulfate) (PtBS-PSS) adsorbed onto hydrophobic and hydrophilic surfaces on their interaction potential were investigated by Li et al. [147] using SFA. It was found that the non-soluble blocks PtBS has a stronger interaction with the hydrophobic surface and, thus, higher adsorbed amount could be achieved. The authors examined the effect of salt concentration, $c_{\text {salt }}$, and polymer molar mass, $M$, on the brush height, $H_{\text {brush }}$. The resulting scaling relationship was found to be in good agreement with predictions of the brush model [148], $H_{\text {brush }} \sim M^{1.0}$ in the low-salt limit and $H_{\text {brush }} M^{-1} \sim\left(c_{\text {salt }} / \sigma_{\text {brush }}\right)^{-0.32}$ in the salted regime, when adsorption took place onto the hydrophobic mica surface. For adsorption on the hydrophilic mica surface, $H_{\text {brush }} M^{-0.7} \sim C_{\text {salt }}^{-0.17}$ agrees with the scaling prediction of the sparse tethering model [148]. The results suggested that, on the hydrophilic bare mica surface, the amount of adsorbed polymer is not high enough to form a brush structure and only very little intermolecular stretching of the attached chains occurs; on the contrary, the hydrophobic surface favours an increased polymer density such that the polyelectrolyte chains adopt a brush conformation.

Interactions between DNA-grafted colloids were measured using optical tweezers by Kegler et al. [98]. Changing the grafting density enabled the authors to trace the transition from the "mushroom"- to the "brush" - regime as shown in figure 17 (see section 2.2. Attached and grafted 
polymers). The measured interaction forces were purely repulsive for all grafting densities. It was found that with decreasing grafting density the force-separation dependence approached that of hard spheres. For small grafting densities the length of the grafted DNA chains did not show an effect on the force-separation dependence, which indicated that the polymers were in the "mushroom"--regime. The interaction in this regime was found to show a scaling with the grafting density which leveled off to the behavior of brushes as it is shown in figure 17. For the latter the transition from an osmotic to a salted brush was traced by the authors in detail by varying the salt concentration in accordance with MF theories [101] (see section 2.2.3. Grafted polymers). At low salt concentration the brush thickness was nearly independent of the salt concentration. The transition from the osmotic to the salted brush was found to take place when the external salt concentration equals the concentration inside the brush. In the salted brush regime the brush thickness shrunk upon addition of salt in accordance with equation (17).

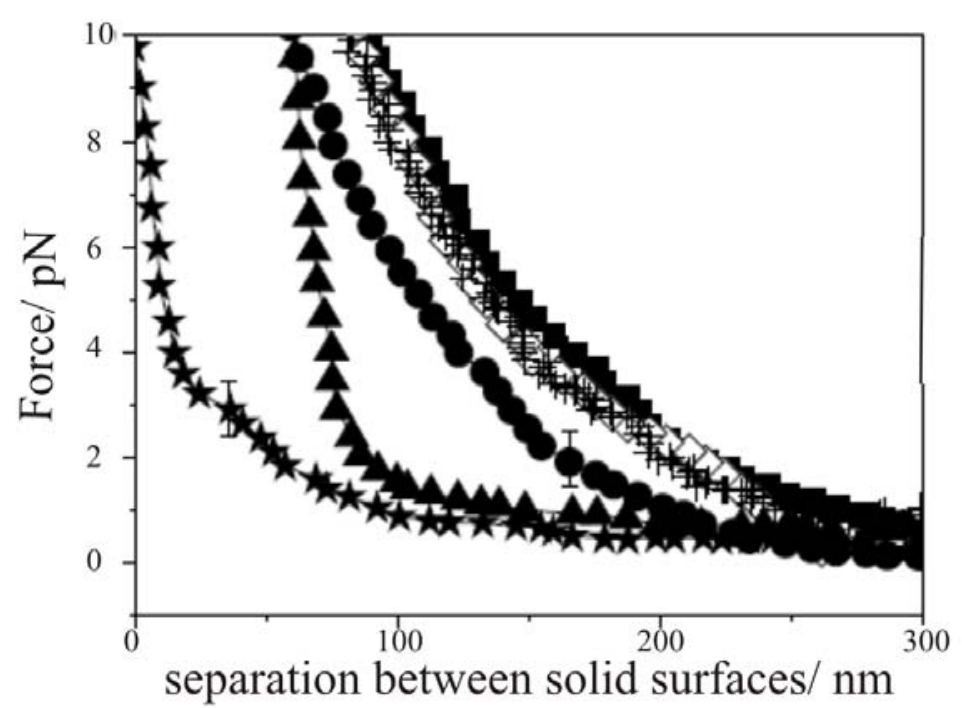

Figure 17. Forces of interaction between DNA-grafted colloids with varying grafting density (匹: $1.84 \cdot 10^{-4}$ chains $/ \mathrm{nm} 2 ;+: 1.51 \cdot 10^{-4}$ chains $/ \mathrm{nm}^{2} ; \square: 8.54 \cdot 10^{-5}$ chains $/ \mathrm{nm}^{2} ; \quad 0: 5.91 \cdot 10^{-5}$ chains $/ \mathrm{nm}^{2} ; \mathbf{\Delta}: 3.95 \cdot 10^{-5}$ chains $/ \mathrm{nm}^{2} ; \star: 1.97 \cdot 10^{-5}$ chains $/ \mathrm{nm}^{2}$ in buffered $\left(10 \mathrm{mM} \mathrm{C} \mathrm{CH}_{11} \mathrm{NO}_{3}\right.$, $\mathrm{pH}$ 8.5) solution. The lines are guides to the eye. Reprinted figure with permission from: Kegler K, Salomo M and Kremer F 2007 Phys. Rev. Lett. 98 058304. Copyright 2007 by the American Physical Society. http://link.aps.org/abstract/PRL/v98/e058304 


\section{Conclusions and outlook}

In this review we attempt to give a description of the progress reached to date in theoretical and experimental work on polymer-induced interactions in colloidal systems. While the first theories date back to the 1950s, experimental measurements first became possible about twenty years later with the invention of the SFA. However only in the last two decades direct and model independent methods, which are sensitive enough to measure the force or potential between colloids became available with AFM, optical tweezers and TIRM. Especially the two latter are non-invasive techniques and extremely sensitive, which enables to test new theories on the pair interaction level even for very weak interaction in the sub- $k_{B} T$ range.

The theoretical models for polymer-induced interaction nowadays include a wide variety of polymer shapes, solvent conditions and tendencies to adsorption. On the other hand, the effect of polymer size polydispersity and charges on colloid and polymer have gained little attention both experimentally and theoretically. Certainly this is a field where further systematic investigations are desirable. Moreover, the dynamic aspect of polymer-induced interactions has not been investigated systematically. For instance, depletion interactions need time to build up through many collisions between the test particles and depletants [149]. Thus, investigations of the short time fluctuations of depletion forces appear to be a promising tool to shed light on the microscopic nature of depletion forces.

So far all TIRM, optical tweezers and AFM-CP studies were performed with spherical probe particle. With the increasing availability of well defined colloids of different shape, it is a challenging perspective to study the pair interaction potential between non-spherical objects (e.g. rod-like). This is particularly, interesting in the experimental field, as there are already some analytical theories describing, f.i., depletion interaction between colloids of anisotropic shape in solution of ideal polymer chains [150-152].

\section{Appendix. List of direct experimental findings on polymer-induced interactions}


In addition to the experimental findings which we discussed in section 4 in detail, we are listing experimental results, without any comments in two tables below. These tables are meant as a reference list, which should enable the reader to quickly look up the most qualitative outcome of experiments on a given system. Therefore the tables are ordered according to the nature of the probe colloids first and second with respect to the polymer solvent system.

In table 1 we are listing experiments where the probe surfaces or particles were immersed in a solution of polymer and any adsorption of the polymer onto the particle interface occurred under experimental conditions. Differently, in the experiments we list in table 2, the polymers were grafted or physically adsorbed onto the probe surfaces, before the actual force measuring experiment. 
Table 1. Forces between colloids in presence of polymer solutions

\begin{tabular}{|c|c|c|c|c|}
\hline \multicolumn{2}{|r|}{ System } & \multirow[t]{2}{*}{ Method } & \multirow[t]{2}{*}{ Results } & \multirow[t]{2}{*}{ Reference } \\
\hline 'Colloids' & Polymer solution & & & \\
\hline Mica & $\begin{array}{c}\text { PS/ toluene } \\
6 \cdot 10^{5} \mathrm{~g} / \mathrm{mol}, 3 \cdot 10^{6} \mathrm{~g} / \mathrm{mol}\end{array}$ & SFA & Out of the sensitivity limit of SFA & {$[122]$} \\
\hline $\begin{array}{l}\text { Mica with adsorbed Triton } \\
\text { X-100 chains to prevent } \\
\text { adsorption of PEO }\end{array}$ & $\begin{array}{c}\mathrm{PEO} / \text { water } \\
3.8 \cdot 10^{4} \mathrm{~g} / \mathrm{mol}, \mathrm{M}_{\mathrm{w}} / \mathrm{M}_{\mathrm{n}}=1.4 \\
4 \cdot 10^{4} \mathrm{~g} / \mathrm{mol}, \mathrm{M}_{\mathrm{w}} / \mathrm{M}_{\mathrm{n}}=1.03\end{array}$ & SFA & $\begin{array}{l}\text { Steric repulsion due to PEO chains adsorption } \\
\text { on mica }\end{array}$ & [124] \\
\hline Mica & $\begin{array}{c}\text { NaPSS } / \text { water } \\
6.5 \cdot 10^{3}--6.9 \cdot 10^{6} \mathrm{~g} / \mathrm{mol}, \mathrm{M}_{\mathrm{w}} / \mathrm{M}_{\mathrm{n}}=1.1\end{array}$ & SFA & $\begin{array}{l}\text { Depletion due to NaPSS in rod-like } \\
\text { conformation }\end{array}$ & [153] \\
\hline Mica & CTAB/ water & SFA & $\begin{array}{c}\text { Depletion; oscillatory potential due to packing } \\
\text { of charged micelles }\end{array}$ & {$[120,133]$} \\
\hline $\begin{array}{l}\mathrm{SiO}_{2}-\mathrm{C}_{18} \text { sphere } \\
\text { with } a=3.8 \mu \mathrm{m}\end{array}$ & $\begin{array}{c}\text { PDMS/ cyclohexane } \\
1.2 \cdot 10^{5} \mathrm{~g} / \mathrm{mol}, \mathrm{M}_{\mathrm{w}} / \mathrm{M}_{\mathrm{n}}=2.3\end{array}$ & AFM & $\begin{array}{c}\text { Depletion; } \\
\delta=10 \mathrm{~nm} \sim R_{g}\end{array}$ & [121] \\
\hline $\begin{array}{l}\mathrm{SiO}_{2}-\mathrm{C}_{18} \text { sphere } \\
\text { with } a=3.0 \mu \mathrm{m}\end{array}$ & $\begin{array}{c}\text { PDMS/ cyclohexane } \\
1.4 \cdot 10^{4} \mathrm{~g} / \mathrm{mol}, 3.1 \cdot 10^{4} \mathrm{~g} / \mathrm{mol} \\
8.3 \cdot 10^{4} \mathrm{~g} / \mathrm{mol}, 1.2 \cdot 10^{5} \mathrm{~g} / \mathrm{mol}\end{array}$ & AFM & Depletion, $\delta$ decreases with increasing $c_{p}$ & [154] \\
\hline $\begin{array}{c}\mathrm{SiO}_{2} \\
\text { with } a=3.5 \mu \mathrm{m}\end{array}$ & $\begin{array}{c}\mathrm{NaPSS} / \text { water } \\
6.8 \cdot 10^{3} \mathrm{~g} / \mathrm{mol}, 3.4 \cdot 10^{4} \mathrm{~g} / \mathrm{mol} \\
7.7 \cdot 10^{4} \mathrm{~g} / \mathrm{mol}, 6.5 \cdot 10^{5} \mathrm{~g} / \mathrm{mol} \\
\mathrm{M}_{\mathrm{w}} / \mathrm{M}_{\mathrm{n}}=1.1\end{array}$ & AFM & Depletion; fitting with equation (1) & [155] \\
\hline $\mathrm{SiO}_{2}$ & $\begin{array}{c}\text { NaPSS/ water } \\
4.6 \cdot 10^{4} \mathrm{~g} / \mathrm{mol}, 2 \cdot 10^{5} \mathrm{~g} / \mathrm{mol}\end{array}$ & AFM & $\begin{array}{l}\text { Depletion; oscillatory potential due to packing } \\
\text { of charged polymers }\end{array}$ & {$[156]$} \\
\hline
\end{tabular}




\begin{tabular}{|c|c|c|c|c|}
\hline $\begin{array}{l}\mathrm{SiO}_{2} \text { sphere } \\
\text { with } a=4.5 \mu \mathrm{m}\end{array}$ & $\begin{array}{l}\text { Polyacrilic acid (PAA)/ water } \\
1.1 \cdot 10^{5} \mathrm{~g} / \mathrm{mol}, \mathrm{M}_{\mathrm{w}} / \mathrm{M}_{\mathrm{n}}=1.13\end{array}$ & AFM & $\begin{array}{c}\text { Depletion; oscillatory potential due to packing } \\
\text { of charged polymers }\end{array}$ & {$[157,158]$} \\
\hline $\begin{array}{c}\mathrm{SiO}_{2} \text { sphere } \\
\text { with } a=2.5 \mu \mathrm{m}\end{array}$ & $\begin{array}{l}\text { Pluronic F 108, SDS/ water } \\
\qquad 1.5 \cdot 10^{4} \mathrm{~g} / \mathrm{mol}\end{array}$ & AFM & $\begin{array}{c}\text { Depletion due to large, charged polymer- } \\
\text { surfactant complexes }\end{array}$ & [159] \\
\hline $\begin{array}{l}\mathrm{SiO}_{2} \text { sphere } \\
\text { with } a=1.8 \mu \mathrm{m}\end{array}$ & $\begin{array}{l}\mathrm{SiO}_{2} \text { nanospheres, } a=11 \mathrm{~nm} / \text { water } \\
\text { PS nanospheres/ water } \\
a=11 \mathrm{~nm} \text { and } 16 \mathrm{~nm}\end{array}$ & AFM & $\begin{array}{c}\text { Study on influence of polydispersity of } \\
\text { macromolecular size and surface charge on the } \\
\text { depletion interaction }\end{array}$ & {$[137]$} \\
\hline $\mathrm{SiO}_{2}-\mathrm{C}_{18}$ sphere & $\begin{array}{l}\text { Bisurea 2,4-bis(2-ethylhexylureido)toluene } \\
\text { (EHUT)/ cyclohexane } \\
\text { Stopper: Monofunctional monomer 2,4- } \\
\text { bis(dibutylureido)toluene (DBUT) }\end{array}$ & AFM & $\begin{array}{l}\text { Depletion, fitting with equation (1); } \\
\text { tuned interaction by adding monofunctional } \\
\text { chain stoppers to the solution }\end{array}$ & [160] \\
\hline $\begin{array}{l}\mathrm{SiO}_{2} \text { spheres } \\
\text { with } a=0.5 \mu \mathrm{m}\end{array}$ & Fd-rods/ water & $\begin{array}{l}\text { Optical } \\
\text { tweezers }\end{array}$ & $\begin{array}{l}\text { Depletion; possible to fit with equation (14) if } \\
\text { rods flexibility is taken into account }\end{array}$ & [139] \\
\hline $\begin{array}{l}\mathrm{SiO}_{2} \text { spheres } \\
\text { with } a=0.6 \mu \mathrm{m}\end{array}$ & $\begin{array}{l}\text { DNA/ water } \\
\mathrm{R}_{\mathrm{g}}=500 \mathrm{~nm}\end{array}$ & $\begin{array}{l}\text { Optical } \\
\text { tweezers }\end{array}$ & $\begin{array}{c}\text { Depletion, accounting for excluded volume } \\
\text { interactions gives good agreement with } \\
\text { experiments }\end{array}$ & {$[116,138]$} \\
\hline $\begin{array}{l}\text { Borosilicate glass sphere } \\
\qquad \text { with } a=5 \mu \mathrm{m}\end{array}$ & $\begin{array}{c}\text { NaPSS/ water } \\
3.5 \cdot 10^{5} \mathrm{~g} / \mathrm{mol} \mathrm{M}_{\mathrm{w}} / \mathrm{M}_{\mathrm{n}}=1.01\end{array}$ & TIRM & $\begin{array}{l}\text { Depletion; oscillatory potential due to packing } \\
\text { of charged polymers }\end{array}$ & [161] \\
\hline $\begin{array}{l}\text { Borosilicate glass sphere } \\
\qquad \text { with } a=5 \mu \mathrm{m}\end{array}$ & $\begin{array}{c}\text { NaPSS/ water } \\
3.5 \cdot 10^{5} \mathrm{~g} / \mathrm{mol}, \mathrm{M}_{\mathrm{w}} / \mathrm{M}_{\mathrm{n}}=1.01\end{array}$ & $\begin{array}{l}\text { TIRM } \\
\text { AFM }\end{array}$ & $\begin{array}{c}\text { Depletion; undulation of structural forces were } \\
\text { used to calibrate AFM }\end{array}$ & [135] \\
\hline Silicon nitride $\left(\mathrm{Si}_{3} \mathrm{~N}_{4}\right)$ tip & PAA/ water & AFM & $\begin{array}{c}\text { Depletion; oscillatory potential due to packing } \\
\text { of charged polymers }\end{array}$ & {$[162,163]$} \\
\hline $\begin{array}{c}\text { PS sphere } \\
\text { with } a=1.5 \text { and } 3.0 \mu \mathrm{m}\end{array}$ & $\begin{array}{c}\mathrm{PEO} / \text { water } \\
1 \cdot 10^{6} \mathrm{~g} / \mathrm{mol} \mathrm{M}_{\mathrm{w}} / \mathrm{M}_{\mathrm{n}}=1.07\end{array}$ & TIRM & $\begin{array}{l}\text { Steric repulsion due to PEO chains adsorption } \\
\text { on glass and PEO }\end{array}$ & [132] \\
\hline
\end{tabular}




\begin{tabular}{|c|c|c|c|c|}
\hline $\begin{array}{l}\text { PS sphere } \\
\text { with } a=7.5 \mu \mathrm{m}\end{array}$ & $\begin{array}{l}\mathrm{NaPSS} / \text { water } \\
1.4 \cdot 10^{5} \mathrm{~g} / \mathrm{mol}\end{array}$ & TIRM & $\begin{array}{c}\text { Depletion; structural forces due to packing of } \\
\text { charged polymers }\end{array}$ & {$[164]$} \\
\hline PS sphere with $a=7.5 \mu \mathrm{m}$ & $\mathrm{SiO}_{2}$ nanospheres $(a=6 \mathrm{~nm}) /$ water & TIRM & Depletion and structural forces & {$[165]$} \\
\hline PS sphere with $a=7.5 \mu \mathrm{m}$ & CTAB/ water & TIRM & Depletion and structural forces & {$[134]$} \\
\hline $\begin{array}{c}\text { PS sphere } \\
\text { with } a=2.9 \mu \mathrm{m}\end{array}$ & $\begin{array}{c}\text { Dextran } / \text { water } \\
2.7 \cdot 10^{6} \mathrm{~g} / \mathrm{mol}, \mathrm{M}_{\mathrm{w}} / \mathrm{M}_{\mathrm{n}}=5.6\end{array}$ & TIRM & $\begin{array}{l}\text { Depletion; } \\
\text { strong polydispersity effect on depletion }\end{array}$ & {$[62]$} \\
\hline PS sphere with $a=1.5 \mu \mathrm{m}$ & Fd-virus/ water & TIRM & Depletion; rod flexibility effect & {$[166]$} \\
\hline PS-DVB sphere, $a=1.9 \mu \mathrm{m}$ & Boehmite rods/ water & TIRM & $\begin{array}{c}\text { Depletion; fitting with density functional } \\
\text { theory }\end{array}$ & {$[48,167]$} \\
\hline $\begin{array}{l}\text { PMMA sphere } \\
\text { with } a=0.6 \mu \mathrm{m}\end{array}$ & $\mathrm{SiO}_{2}$ nanospheres $(a=40 \mathrm{~nm}) /$ water & $\begin{array}{l}\text { Optical } \\
\text { tweezers }\end{array}$ & Depletion and structural forces & {$[168]$} \\
\hline $\begin{array}{c}\text { Lipid bilayers } \\
\text { DPPE and DMPC }\end{array}$ & $\begin{array}{c}\mathrm{PEO} / \text { water } \\
1 \cdot 10^{3}--2 \cdot 10^{4} \mathrm{~g} / \mathrm{mol}\end{array}$ & SFA & $\begin{array}{c}\text { Depletion for } 8 \cdot 10^{3} \mathrm{PEO}, \text { for } M_{w}>1 \cdot 10^{4} \text { steric } \\
\text { repulsion }\end{array}$ & {$[125-127]$} \\
\hline
\end{tabular}

Table 2. Forces between colloids with attached and grafted polymers

\begin{tabular}{|c|c|c|c|}
\hline \multicolumn{2}{|c|}{ System } & Method & Results \\
\hline 'Colloid' & Polymer solution & SFA & Steric repulsion due to physically adsorbed \\
\hline Mica & $\mathrm{PEO} /$ toluene & PEO \\
& $4 \cdot 10^{4} \mathrm{~g} / \mathrm{mol}, 1.6 \cdot 10^{5} \mathrm{~g} / \mathrm{mol}$ & Range: $8.5 \pm 1 R_{g}$ \\
& $3.1 \cdot 10^{5} \mathrm{~g} / \mathrm{mol}$ & & \\
& $M_{w} / M_{n}<1.13$ & SFA & Steric repulsion due to physically adsorbed \\
\hline Mica & PEO/water & {$[170]$} \\
\hline
\end{tabular}




\begin{tabular}{|c|c|c|c|c|}
\hline & $1.5 \cdot 10^{5} \mathrm{~g} / \mathrm{mol}$ & & PEO & \\
\hline Mica & $\begin{array}{c}\mathrm{PEO} / \text { water } \\
4 \cdot 10^{4} \mathrm{~g} / \mathrm{mol}, M_{w} / M_{n}=1.04 \\
1.6 \cdot 10^{5} \mathrm{~g} / \mathrm{mol}, M_{w} / M_{n}=1.03\end{array}$ & SFA & $\begin{array}{l}\text { Steric repulsion increasing monotonically on } \\
\text { approach; range } \sim 6 \pm 1 R_{g}\end{array}$ & $\begin{array}{c}{[128,169,} \\
171]\end{array}$ \\
\hline Mica & $\begin{array}{c}\mathrm{PEO} / \text { water } \\
1.2 \cdot 10^{6} \mathrm{~g} / \mathrm{mol}, M_{w} / M_{n}=1.12\end{array}$ & SFA & $\begin{array}{l}\text { At low polymer adsorbance a long-range }(\sim 2.5 \\
\left.\qquad R_{\mathrm{g}}\right) \text { attraction -- bridging; } \\
\text { at full adsorption steric repulsion }\end{array}$ & [145] \\
\hline Mica & Polylysine $\left(9 \cdot 10^{4} \mathrm{~g} / \mathrm{mol}\right) /$ water & SFA & Electrostatic + steric repulsion & {$[172]$} \\
\hline Mica & $\begin{array}{l}\text { PS/ cyclohexane (bad solvent) } \\
6 \cdot 10^{5} \mathrm{~g} / \mathrm{mol}, 9 \cdot 10^{6} \mathrm{~g} / \mathrm{mol}\end{array}$ & SFA & $\begin{array}{l}\text { Attraction at } 4.6 \mathrm{~nm}<h \leq 30 \mathrm{~nm} ; \\
\text { repulsion at } h<4.6 \mathrm{~nm} \text { repulsion }\end{array}$ & {$[173]$} \\
\hline Mica & $\begin{array}{l}\text { PS/ cyclohexane (bad solvent) } \\
6 \cdot 10^{5} \mathrm{~g} / \mathrm{mol}\end{array}$ & SFA & $\begin{array}{c}\text { Attraction at } R_{g}<h \leq 3 \cdot R_{g} ; \\
\text { repulsion at } h<R_{g}\end{array}$ & {$[106]$} \\
\hline Mica & $\begin{array}{c}\text { PS/ cyclopentane (bad solvent) } \\
1.2 \cdot 10^{5} \mathrm{~g} / \mathrm{mol}, 4.9 \cdot 10^{5} \mathrm{~g} / \mathrm{mol} \\
5.2 \cdot 10^{5} \mathrm{~g} / \mathrm{mol}, 1.1 \cdot 10^{6} \mathrm{~g} / \mathrm{mol} \\
M_{w} / M_{n}<1.09\end{array}$ & SFA & $\begin{array}{l}\text { Forces sensitive to solvent quality and solvent } \\
\qquad \text { composition; } \\
\text { attraction at } \mathrm{T}<\mathrm{T}_{\theta}\end{array}$ & {$[174,175]$} \\
\hline Mica & $\begin{array}{c}\text { PS/ cyclopentane } \\
6 \cdot 10^{5} \mathrm{~g} / \mathrm{mol}, 2 \cdot 10^{6} \mathrm{~g} / \mathrm{mol}\end{array}$ & SFA & $\begin{array}{l}\text { Bridging at partial adsorption; } \\
\text { steric repulsion at full adsorption }\end{array}$ & {$[176]$} \\
\hline Mica & $\begin{array}{c}\text { PS/ cyclopentane (near } \theta \text {-solvent) } \\
2 \cdot 10^{5} \mathrm{~g} / \mathrm{mol}, 4 \cdot 10^{5} \mathrm{~g} / \mathrm{mol}, 6.5 \cdot 10^{5} \mathrm{~g} / \mathrm{mol}\end{array}$ & SFA & $\begin{array}{c}\text { Bridging }\left(\text { range } \sim 2.5 R_{\mathrm{g}}\right) ; \\
\text { weaker bridging with increasing } M_{P S}\end{array}$ & {$[177]$} \\
\hline Mica & $\begin{array}{l}\text { Ethyl-(hydroxyethy1)cellulose (EHEC)/ } \\
\text { water (bad solvent) }\end{array}$ & SFA & $\begin{array}{c}\text { Forces sensitive to } \mathrm{T} ; \\
\text { ambient T: purely repulsive; } \\
\text { above the cloud point: repulsive but less long- }\end{array}$ & {$[178]$} \\
\hline
\end{tabular}




\begin{tabular}{|c|c|c|c|c|}
\hline & & & $\begin{array}{c}\text { ranged, due to contraction of the EHEC layer in } \\
\text { the bad solvent }\end{array}$ & \\
\hline Mica & $\begin{array}{l}\text { Poly }(\alpha \text {-methylstyrene })(\mathrm{P} \alpha \mathrm{MS}) / \\
\text { cyclohexane } \\
9 \cdot 10^{4} \mathrm{~g} / \mathrm{mol}, M_{w} / M_{n}<1.1\end{array}$ & SFA & $\begin{array}{l}\text { Bridging; segmental sticking energy of polymer } \\
\text { to mica } \sim 1 / 3 k_{B} T\end{array}$ & {$[144]$} \\
\hline Mica & $\begin{array}{c}\text { PS-PEO/ toluene, xylene } \\
\text { PS-X (X = sec-butyl, phenil) } \\
\text { a range of } M_{w} \text { of each of the blocks } \\
\qquad M_{w} / M_{n} \leq 1.1\end{array}$ & SFA & $\begin{array}{c}\text { No bridging even at low coverage; } \\
\text { only steric repulsion }\end{array}$ & {$[179-181]$} \\
\hline Mica & $\begin{array}{c}\text { PVP-PI, PVP-PS/ toluene } \\
\text { a range of } M_{w} \text { of each of the blocks }\end{array}$ & SFA & Steric repulsive forces & {$[182]$} \\
\hline Mica & $\begin{array}{l}\text { PVP-PS/ cyclohexane } \\
\text { a range of } M_{w} \text { of each of the blocks }\end{array}$ & SFA & Steric repulsion & {$[183]$} \\
\hline Mica & $\begin{array}{l}\text { PVP-PS, PS-PVP-PS/ cyclohexane }(\sim \theta-T) \\
\text { a range of } M_{w} \text { of each of the blocks }\end{array}$ & SFA & $\begin{array}{l}\text { Brush repulsion; } \\
\text { brush described with a MF model [182] }\end{array}$ & {$[184]$} \\
\hline Mica & PEO-lysine/ water & SFA & Electro-steric repulsion & {$[185]$} \\
\hline $\begin{array}{l}\text { Mica, } \\
\text { Hydrophobic, hydrophilic }\end{array}$ & $\begin{array}{c}\text { PtBSP-NaPSS/ water } \\
\text { a range of } M_{w} \text { of each of the blocks }\end{array}$ & SFA & $\begin{array}{l}\text { Brushes formed at hydrophobic surfaces; } \\
\text { Brush repulsion }\end{array}$ & {$[147]$} \\
\hline Mica hydrophobic & PtBMA-b-PGMAS/ water & SFA & Electro-steric repulsion & {$[186]$} \\
\hline $\begin{array}{c}\mathrm{SiO}_{2} \text { spheres } \\
\text { with } a=0.6 \mu \mathrm{m}\end{array}$ & $\begin{array}{c}\mathrm{PEO} / \text { water (good solvent) } \\
4.5 \cdot 10^{5} \mathrm{~g} / \mathrm{mol}, 7.6 \cdot 10^{5} \mathrm{~g} / \mathrm{mol} \\
9.9 \cdot 10^{5} \mathrm{~g} / \mathrm{mol}, 1.6 \cdot 10^{6} \mathrm{~g} / \mathrm{mol} \\
M_{w} / M_{n}<1.09\end{array}$ & $\begin{array}{l}\text { Optical } \\
\text { tweezers }\end{array}$ & $\begin{array}{l}\text { Steric repulsion due to adsorbed PEO; } \\
\text { exponential over the range of energies }\left(0.1 k_{B} T\right. \\
\left.-5 k_{B} T\right)\end{array}$ & {$[141]$} \\
\hline $\mathrm{SiO}_{2}$ sphere & Polyethylene imine (PEI)/ water (good & AFM & Electro-steric repulsion by approach; & {$[143,187]$} \\
\hline
\end{tabular}




\begin{tabular}{|c|c|c|c|c|}
\hline with $a=3.4 \mu \mathrm{m}$ & $\begin{array}{c}\text { solvent }) \\
4 \cdot 10^{3} \mathrm{~g} / \mathrm{mol}, 3 \cdot 10^{4} \mathrm{~g} / \mathrm{mol} \\
3 \cdot 10^{5} \mathrm{~g} / \mathrm{mol}, 5 \cdot 10^{6} \mathrm{~g} / \mathrm{mol}\end{array}$ & & Bridging during retraction & \\
\hline $\mathrm{SiO}_{2}$ sphere & PMMA/ toluene & AFM & $\begin{array}{l}\text { Strong steric repulsion due to dense polymer } \\
\text { brushes }\end{array}$ & {$[188,189]$} \\
\hline $\begin{array}{c}\text { Glass sphere } \\
\text { with } a=60 \mu \mathrm{m}\end{array}$ & $\begin{array}{c}\text { PEO/ water (good solvent) } \\
5.6 \cdot 10^{4} \mathrm{~g} / \mathrm{mol}\end{array}$ & AFM & $\begin{array}{l}\text { Bridging at low surface coverage; } \\
\text { Steric repulsion at full coverage }\end{array}$ & {$[142]$} \\
\hline $\mathrm{Si}_{3} \mathrm{~N}_{4}$ AFM-tip & $\begin{array}{c}\text { Poly } N \text {-vinyl-2-pyrrolidone (PVP)/ } \\
\text { water (good solvent) } \\
1.3 \cdot 10^{6} \mathrm{~g} / \mathrm{mol} \\
\mathrm{SDS}\end{array}$ & AFM & $\begin{array}{l}\text { Charged polymer-surfactant complexes; } \\
\text { enhanced electro-steric repulsion }\end{array}$ & {$[190]$} \\
\hline $\begin{array}{c}\mathrm{Si}_{3} \mathrm{~N}_{4} \text { AFM-tip } \\
\text { Mica }\end{array}$ & $\begin{array}{c}\text { PVP-NaPSS/ toluene } \\
\text { a range of } M_{w} \text { of each of the blocks }\end{array}$ & $\begin{array}{l}\text { AFM } \\
\text { SFA }\end{array}$ & $\begin{array}{c}\text { Steric repulsion with a bimodal distribution of } \\
\text { interaction distances due to brush } \\
\text { heterogeneities }\end{array}$ & [191] \\
\hline $\mathrm{Si}_{3} \mathrm{~N}_{4}$ AFM-tip & $\begin{array}{l}\mathrm{PEO} / \text { water } \\
5 \cdot 10^{3} \mathrm{~g} / \mathrm{mol}\end{array}$ & AFM & Steric repulsion & [192] \\
\hline $\mathrm{Si}_{3} \mathrm{~N}_{4}$ AFM-tip & PS, PEO-PMMA/ cyclohexane, water & AFM & Exponentially decaying steric repulsion & [193] \\
\hline $\begin{array}{c}\mathrm{Si}_{3} \mathrm{~N}_{4} \text { AFM-tip } \\
\text { Mica }\end{array}$ & PtBSP-NaPSS/ water & $\begin{array}{l}\text { AFM } \\
\text { SFA }\end{array}$ & $\begin{array}{c}\text { Electro-steric repulsion with a strong } \\
\text { dependence of interaction distance on } c_{\mathrm{NaCl}}\end{array}$ & [191] \\
\hline Silicon tip & $\begin{array}{c}\text { PVP-PS/ toluene, water } \\
\text { a range of } M_{w} \text { of each of the blocks }\end{array}$ & AFM & $\begin{array}{l}\text { Stretched brush repulsion in toluene; } \\
\text { brush collapse in water }\end{array}$ & [194] \\
\hline $\begin{array}{c}\text { PS sphere } \\
\text { with } a=3 \mu \mathrm{m}\end{array}$ & $\begin{array}{c}\text { PEI/ water (good solvent) } \\
7.5 \cdot 10^{5} \mathrm{~g} / \mathrm{mol} \\
\mathrm{NaPSS}\end{array}$ & TIRM & $\begin{array}{c}\text { For more than one polyelectolyte layer } \\
\text { inhomogeneous potentials with extremely long- } \\
\text { ranged repulsive contributions }\end{array}$ & [195] \\
\hline
\end{tabular}




\begin{tabular}{|c|c|c|c|c|}
\hline & $\begin{array}{c}7 \cdot 10^{4} \mathrm{~g} / \mathrm{mol} \\
\text { Polydiallylamine } \\
\text { Dimethyl-ammonium bromide } \\
(\text { PDADMAC) } \\
1 \cdot 10^{5} \mathrm{~g} / \mathrm{mol}\end{array}$ & & & \\
\hline $\begin{array}{c}\text { PS sphere } \\
\text { with } a=0.33 \mu \mathrm{m}\end{array}$ & $\begin{array}{l}\text { PDMA, PMEA, PNIPAM, PMEA-b- } \\
\text { PNIPAM/ water }\end{array}$ & AFM & $\begin{array}{l}\text { Bridging being dependent on grafting density } \\
\text { and monomer nature }\end{array}$ & [146] \\
\hline $\begin{array}{l}\text { Streptavidin covered } \\
\text { spheres with } a=1 \mu \mathrm{m}\end{array}$ & $\begin{array}{c}\text { DNA/ water } \\
\text { Different grafting density }\end{array}$ & $\begin{array}{l}\text { Optical } \\
\text { tweezers }\end{array}$ & Electro-steric repulsion & [98] \\
\hline $\begin{array}{l}\text { Zirconia sphere } \\
\text { with } a=10 \mu \mathrm{m}\end{array}$ & $\begin{array}{l}\text { PAA/ water } \\
7.5 \cdot 10^{5} \mathrm{~g} / \mathrm{mol}\end{array}$ & AFM & $\begin{array}{l}\text { Bridging at low coverage; } \\
\text { repulsion at high coverage; }\end{array}$ & [196] \\
\hline $\begin{array}{l}\text { Emulsion of } \mathrm{Fe}_{2} \mathrm{O}_{3} \text { in octane } \\
\text { stabilized with oleic acid } \\
\qquad a=100 \mathrm{~nm}\end{array}$ & $\begin{array}{c}\text { Polyvynil alcohol (PVA)/ water } \\
\text { Surfactants: SDS, } \\
\text { CTAB, } \\
\text { tetradecyltrimethylammonium bromide, } \\
\text { nonyl phenol ethoxylate (NP10) }\end{array}$ & $\begin{array}{l}\text { Magnetic } \\
\text { field } \\
\text { induced } \\
\text { chaining } \\
\text { technique } \\
\text { (MCT) }\end{array}$ & $\begin{array}{l}\text { Large polymer-surfactants complexes } \\
\text { generating strong steric repulsion; } \\
\text { no synergetic effect with NP10 }\end{array}$ & [197] \\
\hline
\end{tabular}




\section{References}

[1] Hiemenz P C and Rajagopalan R 1997 Principles of Colloid and Surface Chemistry 3rd ed (New York: Marcel Dekker Inc.) 672

[2] Vingerhoeds M H, Blijdenstein T B J, Zoet F D and Van Aken G A 2005 Food Hydrocolloids 19915

[3] Silletti E, Vingerhoeds M H, Norde W and Van Aken G A 2007 Food Hydrocolloids 21596

[4] Ten Grotenhuis E, Tuinier R and De Kruif C G 2003 J. Dairy Sci. 86764

[5] Gast a P, Hall C K and Russel W B 1983 J. Colloid Interface Sci. 96251

[6] Klein R 2002 Interacting colloidal suspensions, in Neutrons, X-rays and light: scattering methods applied to soft condensed matter, Lindner P and Zemb T, Editors. (Amsterdam: Elsevier). p. 351-381.

[7] Israelachvili J N 1991 Intermolecular and Surface Forces 2nd ed (London: Academic Press)

[8] Furst E M 2003 Soft Materials 1167

[9] Grier D G 1997 Cur. Opinion Colloid Interface Sci. 2264

[10] Molloy J E and Padgett M J 2002 Contemporary Physics 43241

[11] Vossen D L J, Van Der Horst A, Dogterom M and Van Blaaderen A 2004 Rev. Sci. Instrum. 752960

[12] Butt H J, Cappella B and Kappl M 2005 Surface Sci. Rep. 591

[13] Prieve D C 1999 Adv. Colloid Interface Sci. 8293

[14] Walz J Y 1997 Cur. Opinion Colloid Interface Sci. 2600

[15] Dijkstra M, Brader J M and Evans E 1999 J. Phys.: Condens. Matter 1110079

[16] Louis a A 2002 J. Phys.: Condens. Matter 149187

[17] Fleer G J, Cohen Stuart M A, Scheutjens J M H M, Cosgrove T and Vincent B 1993 Polymers at Interfaces (London: Chapman\&Hall) 502

[18] The compilation is, probably, far from being complete and determined by personal bias, which we apologize for.

[19] Netz R R and Andelman D 2003 Phys. Rep. 3801

[20] Derjaguin B V 1934 Kolloid Zeits. 69155

[21] Asakura S and Oosawa F 1954 J. Chem. Phys. 221255

[22] Tuinier R and Fleer G J 2004 Macromolecules 378764

[23] Tuinier R, Vliegenthart G A and Lekkerkerker H N W 2000 J. Chem Phys. 11310768

[24] Asakura S and Oosawa F 1958 J. Polym. Sci. 33183

[25] Vrij A 1976 Pure Appl. Chem. 48471

[26] De Hek H and Vrij A 1981 J. Colloid Interface Sci. 84409

[27] Eisenriegler E 1983 J. Chem. Phys. 791052

[28] Tuinier R, Rieger J and De Kruif C G 2003 Adv. Colloid Interface Sci. 1031

[29] De Gennes P G 1981 Macromolecules 141637 
[30] De Gennes P G 1982 Macromolecules 15492

[31] De Gennes P G 1985 Acad. Sci. Ser. II 300839

[32] Feigin R I and Napper D H 1980 J. Colloid Interface Sci. 75525

[33] Joanny J F, Leibler L and De Gennes P G 1979 J. Polym. Sci. 171073

[34] Scheutjens J M H M and Fleer G J 1982 Adv. Colloid Interface Sci. 16361

[35] De Gennes P G 1979 Scaling Concepts in Polymer Physics (Ithaca: Cornell University Press)

[36] Scheutjens J M H M and Fleer G J 1979 J. Phys. Chem. 831619

[37] Cowell C, Li-in-on R and Vincent B 1978 J. Chem. Soc. Faraday Trans. 74337

[38] De Gennes P G 1979 Comptes Rendus Hebdomadaires Des Seances De L Academie Des Sciences Serie B 288359

[39] Tuinier R, Lekkerkerker H N W and Aarts D G a L 2002 Phys. Rev. E 65060801

[40] Bohmer M R, Evers O A and Scheutjens J M H M 1990 Macromolecules 232288

[41] Fleer G J, Skvortsov a M and Tuinier R 2003 Macromolecules 367857

[42] Tuinier R, Aarts D G a L, Wensink H H and Lekkerkerker H N W 2003 Phys. Chem. Chem. Phys. 53707

[43] Hall D G 1972 J. Chem. Soc. Faraday Trans. 682169

[44] Bolhuis P G, Louis a A, Hansen J P and Meijer E J 2001 J. Chem. Phys. 1144296

[45] Pelissetto A and Hansen J P 2006 Macromolecules 399571

[46] Roth R, Evans E and Dietrich S 2000 Phys. Rev. E 625360

[47] Bechinger C, Rudhardt D, Leiderer P, Roth R and Dietrich S 1999 Phys. Rev. Lett. 833960

[48] Helden L, Roth R, Koenderink G H, Leiderer P and Bechinger C 2003 Phys. Rev. Lett. 90 048301

[49] Ferreira P G, Dymitrowska M and Belloni L 2000 J. Chem. Phys. 1139849

[50] Vincent B, Edwards J, Emmett S and Jones A 1986 Colloids Surf. 18261

[51] Wijmans C M, Zhulina E B and Fleer G J 1994 Macromolecules 273238

[52] Striolo A 2006 Phys. Rev. E 74041401

[53] Broukhno A, Jonsson B, Akesson T and Vorontsov-Velyaminov P N 2000 J. Chem. Phys. 113 5493

[54] Mao Y 1995 J. Phys. 51761

[55] Chu X L, Nikolov a D and Wasan D T 1996 Langmuir 125004

[56] Goulding D and Hansen J P 2001 Molecular Physics 99865

[57] Piech M and Walz J Y 2000 J. Colloid Interface Sci. 225134

[58] Sear R P and Frenkel D 1997 Phys. Rev. E. 551677

[59] Walz J Y 1996 J. Colloid Interface Sci. 178505

[60] Warren P B 1997 Langmuir 134588

[61] Tuinier R and Petukhov a V 2002 Macromol. Theory Simul. 11975

[62] Kleshchanok D, Tuinier R and Lang P R 2006 Langmuir 229121 
[63] Boyd R H and Phillips P J 1993 The science of polymer molecules (Cambridge: Cambridge University Press) p.34

[64] Croze O A and Cates M E 2005 Langmuir 215627

[65] Auvray L 1981 J. Phys. 4279

[66] Mao Y, Cates M E and Lekkerkerker H N W 1995 Phys. Rev. Lett. 754548

[67] Mao Y, Cates M E and Lekkerkerker H N W 1996 J. Phys. Chem. 1063721

[68] Eisenriegler E 2006 J. Chem Phys. 125204903

[69] De Vries R 2006 J. Chem. Phys. 125014905

[70] Yaman K, Jeppesen C and Marques C M 1998 Europhys. Lett. 42221

[71] Jenkel E and Rumbach B 1951 Z. Elektrochem. 55612

[72] De Gennes P G 1976 J. Physique 371443

[73] Naji A, Seidel C and Netz R R 2006 Adv. Polym. Sci. 198149

[74] Netz R R and Andelman D 2003 Phys. Rep. 3801 - 95

[75] De Gennes P G 1982 Maromolecules 15492 - 500

[76] Scheutjens J M H M and Fleer G J 1985 Macromolecules 181882 - 1900

[77] Semenov a N 1996 J. Phys. II 61759 - 1780

[78] Klein J and Rossi J 1998 Macromolecules 311979 - 1988

[79] Klein J and Pincus P A 1982 Macromolecules 151129 - 1135

[80] Klein J 1980 Nature (London) 288248 - 250

[81] Klein J and Luckham P F 1984 Nature (London) 308836 - 837

[82] Klein J and Luckham P F 1982 Nature (London) 300429 - 431

[83] Israelachvili J N, Tandor R K and White L R 1979 Nature (London) 277120 - 121

[84] Napper D H 1983 Polymeric Stabilization of Colloidal Dispersions (London: Academic Press)

[85] Bhatia S R and Russel W B 2000 Macromolecules 335713

[86] Dolan a K and Edwards S F 1974 Proc. R. Soc. London 337509

[87] Porte G, Ligoure C, Appell J and Aznar R 2006 J. Stat. Mech. 05 P05005

[88] Cao D and Wu J 2006 Langmuir 222712

[89] Alexander S 1977 J. Phys. 38983 - 987

[90] De Gennes P G 1985 Acad. Sci. Ser. II, Fascicule B Mechanique Physique Chimie Astronomie 300839 - 843

[91] Zhulina E B, Borisov O V and Priamitsyn V A 1990 J. Colloid Interface Sci. 137495 - 511

[92] Cosgrove T, Heath T, Van Lent B, Leermakers F a M and Scheutjens J M H M 1987 Macromolecules 201692

[93] Milner S T, Witten T A and Cates M E 1988 Macromolecules 212610

[94] Manciu M and Ruckenstein E 2004 Langmuir 206490

[95] Miklavic S J and Marcelja S 1988 J. Phys. Chem. 926718

[96] Misra S, Varanasi S and Varanasi P P 1989 Macromolecules 224173 
[97] Pincus P 1991 Macromolecules 242912

[98] Kegler K, Salomo M and Kremer F 2007 Phys. Rev. Lett. 98058304

[99] Linse P 2007 J. Chem Phys. 126114903

[100] Tamashiro M N, Hernandez-Zapata E, Schorr P A, Balastre M, Tirrell M and Pincus P $2001 \mathrm{~J}$. Chem. Phys. 1151960

[101] Balastre M, Li F, Schorr P, Yang J C, Mays J W and Tirrell M V 2002 Macromolecules 35 9480

[102] Milner S T, Witten T A and Cates M E 1989 Macromolecules 22853

[103] Tabor D and Winterton R H S 1968 Nature 2191120

[104] Israelachvili J N and Adams G E 1976 Nature 262774

[105] Israelachvili J N and Adams G E 1978 J. Chem. Soc. Faraday Trans. 74975

[106] Klein J 1980 Nature (London) 288248

[107] Binnig G, Quate C F and Gerber C 1986 Phys. Rev. Lett. 56930

[108] Ralston J, Larson I, Rutland M W, Feiler a A and Kleijn M 2005 Pure Appl. Chem. 772149

[109] Ducker W A, Senden T J and Pashley R M 1991 Nature 353239

[110] Prieve D C and Walz J Y 1993 Appl. Opt. 321629

[111] Helden L, Eremina E, Riefler N, Hertlein C, Bechinger C, Eremin Y and Wriedt T 2006 Appl. Opt. 457299

[112] Ashkin A 1970 Phys. Rev. Lett. 24156

[113] Schmidt C F 2006 A practical guide to optical tweezers, in Soft condensed matter physics in molecular and cell biology, Poon W C K and Andelman D, Editors. (New York, London: Taylor\&Francis). p. 279.

[114] Svoboda K and Block S M 1994 Annu. Rev. Biophys. Biomol. Struct. 23247

[115] Crocker J C and Grier D G 1996 J. Colloid Interface Sci. 179298

[116] Verma R, Crocker J C, Lubensky T C and Yodh a G 2000 Macromolecules 33177

[117] Allersma M A, Gittes F, De Castro M J, Stewart R J and Schmidt C F 1998 Biophys. J. 74 1074

[118] Han Y L and Grier D G 2003 Phys. Rev. Lett. 91038302

[119] Baumgartl J and Bechinger C 2005 Europhys. Lett. 71487

[120] Richetti P and Kekicheff P 1992 Phys. Rev. Lett. 681951

[121] Milling a J and Biggs S 1995 J. Colloid Interface Sci. 170604

[122] Luckham P F and Klein J 1985 Macromolecules 18721

[123] Van Der Beek G P, Cohen Stuart M A, Fleer G J and Hofman J E 1989 Langmuir 51180

[124] Luckham P F and Klein J 1987 J. Colloid Interface Sci. 117149

[125] Kuhl T, Guo Y Q, Alderfer J L, Berman a D, Leckband D, Israelachvili J and Hui S W 1996 Langmuir 123003

[126] Kuhl T L, Berman a D, Hui S W and Israelachvili J N 1998 Macromolecules 318250 
[127] Kuhl T L, Berman a D, Hui S W and Israelachvili J N 1998 Macromolecules 318258

[128] Klein J and Luckham P F 1984 Macromolecules 171041

[129] Rudhardt D, Bechinger C and Leiderer P 1998 Phys. Rev. Lett. 1330

[130] Rudhardt D, Bechinger C and Leiderer P 1999 J. Phys.: Condens. Matter 1110073

[131] Ohshima Y N, Sakagami H, Okumoto K, Tokoyoda A, Igarashi T, Shintaku K B, Toride S, Sekino H, Kabuto K and Nishio I 1997 Phys. Rev. Lett. 783963

[132] Kleshchanok D and Lang P 2007 Langmuir 234332

[133] Kekicheff P, Nallet F and Richetti P 1994 J. Physique 4735

[134] Sober D L and Walz J Y 1995 Langmuir 112352

[135] Biggs S, Prieve D C and Dagastine R R 2005 Langmuir 215421

[136] Jönsson B, Broukhno A, Forstman J and Akesson T 2003 Langmuir 199914

[137] Piech M and Walz J Y 2002 J. Colloid Interface Sci. 253117

[138] Verma R, Crocker J C, Lubensky T C and Yodh a G 1998 Phys. Rev. Lett. 814004

[139] Lin K, Crocker J C, Zeri a C and Yodh a G 2001 Phys. Rev. Lett. 87088301

[140] Lau a W C, Lin K H and Yodh a G 2002 Phys. Rev. E 66020401

[141] Owen R J, Crocker J C, Verma R and Yodh a G 2001 Phys. Rev. E 64011401

[142] Braithwaite G J C, Howe A and Luckham P F 1996 Langmuir 124224

[143] Pericet-Camara R, Papastavrou G, Behrens S H, Helm C A and Borkovec M 2006 J. Colloid Interface Sci. 296496

[144] Granick S, Patel S and Tirrell M 1986 J. Chem. Phys. 855370

[145] Klein J and Luckham P F 1984 Nature 308836

[146] Goodman D, Kizhakkedathu J N and Brooks D E 2004 Langmuir 202333

[147] Li F, Balastre M, Schorr P, Argillier J F, Yang J C, Mays J W and Tirrell M 2006 Langmuir 224084

[148] Borisov O V, Zhulina E B and Birshtein T M 1994 Macromolecules 274795

[149] Vliegenthart G A and Van Der Schoot P 2003 Europhys. Lett. 62600

[150] Eisenriegler E 2006 Journal of Chemical Physics 125

[151] Eisenriegler E 2006 J. Chem. Phys. 124144912

[152] Eisenriegler E and Bringer A 2005 J. Phys.: Condens. Matter 17 S1711

[153] Marra J and Hair M L 1989 J. Colloid Interface Sci. 128511

[154] Wijting W K, Knoben W, Besseling N a M, Leermakers F a M and Cohen-Stuart M A 2004 Phys. Chem. Chem. Phys. 64432

[155] Biggs S, Burns J L, Yan Y D, Jameson G J and Jenkins P 2000 Langmuir 169242

[156] Milling a J 1996 J. Phys. Chem. 1008986

[157] Milling a J and Vincent B 1997 J. Chem. Soc. Faraday Trans. 933179

[158] Milling a J and Kendall K 2000 Langmuir 165106

[159] Tulpar A, Tilton R D and Walz J Y 2007 Langmuir 234351 
[160] Knoben W, Besseling N a M and Cohen-Stuart M A 2006 Phys. Rev. Lett. 97068301

[161] Biggs S, Dagastine R R and Prieve D C 2002 J. Phys. Chem. 10611557

[162] Burns J L, Yan Y D, Jameson G J and Biggs S 2000 Colloids Surf. 162265

[163] Burns J L, Yan Y D, Jameson G J and Biggs S 2002 J. Colloid Interface Sci. 24724

[164] Sharma A, Tan S N and Walz J Y 1997 J. Colloid Interface Sci. 191236

[165] Sharma A and Walz J Y 1996 J. Chem. Soc. Faraday Trans. 924997

[166] Holmqvist P, Kleshchanok D and Lang P R 2007 Langmuir accepted

[167] Helden L, Koenderink G H, Leiderer P and Bechinger C 2004 Langmuir 205662

[168] Crocker J C, Matteo J A, Dinsmore a D and Yodh a G 1999 Phys. Rev. Lett. 824352

[169] Klein J and Luckham P F 1986 Macromolecules 192007

[170] Israelachvili J N, Tandon R K and White L R 1979 Nature 277120

[171] Klein J and Luckham P F 1982 Nature (London) 300429

[172] Luckham P F and Klein J 1984 J. Chem. Soc. Faraday Trans. 80865

[173] Israelachvili J N, Tirrell M, Klein J and Almog Y 1984 Macromolecules 17204

[174] Hu H W and Granick S 1990 Macromolecules 23613

[175] Hu H W, Vanalsten J and Granick S 1989 Langmuir 5270

[176] Almog Y and Klein J 1985 J. Colloid Interface Sci. 10633

[177] Ruths M, Israelachvili J N and Ploehn H J 1997 Macromolecules 303329

[178] Malmsten M, Claesson P M, Pezron E and Pezron I 1990 Langmuir 61572

[179] Taunton H J, Toprakcioglu C, Fetters L J and Klein J 1988 Nature 332712

[180] Taunton H J, Toprakcioglu C, Fetters L J and Klein J 1988 Nature 332712

[181] Taunton H J, Toprakcioglu C, Fetters L J and Klein J 1990 Macromolecules 23571

[182] Watanabe H and Tirrell M 1993 Macromolecules 266455

[183] Hadziioannou G, Patel S, Granick S and Tirrell M 1986 J. Am. Chem. Soc. 1082869

[184] Kilbey S M, Watanabe H and Tirrell M 2001 Macromolecules 345249

[185] Claesson P M and Golander C G 1987 J. Colloid Interface Sci. 117366

[186] Abraham T, Giasson S, Gohy J F and Jerome R 2000 Langmuir 164286

[187] Papastavrou G, Kirwan L J and Borkovec M 2006 Langmuir 2210880

[188] Yamamoto S, Ejaz M, Tsujii Y and Fukuda T 2000 Macromolecules 335608

[189] Yamamoto S, Ejaz M, Tsujii Y, Matsumoto M and Fukuda T 2000 Macromolecules 335602

[190] Fleming B D, Wanless E J and Biggs S 1999 Langmuir 158719

[191] Kelley T W, Schorr P A, Johnson K D, Tirrell M and Frisbie C D 1998 Macromolecules 31 4297

[192] Nnebe I M and Schneider J W 2006 Macromolecules 393616

[193] Butt H J, Kappl M, Mueller H, Raiteri R, Meyer W and Ruhe J 1999 Langmuir 152559

[194] Overney R M, Leta D P, Pictroski C F, Rafailovich M H, Liu Y, Quinn J, Sokolov J, Eisenberg A and Overney G 1996 Phys. Rev. Lett. 761272 
[195] Kleshchanok D, Wong J E, Von Klitzing R and Lang P R 2006 Progr Colloid Polym. Sci. 133 52

[196] Biggs S 1995 Langmuir 11156

[197] Philip J, Prakash G G, Jaykumar T, Kalyanasundaram P, Mondain-Monval O and Raj B 2002 Langmuir 184625 